\title{
Uniqueness for unbounded solutions to stationary viscous Hamilton-Jacobi equations
}

\author{
Guy Barles And Alessio Porretta
}

\begin{abstract}
We consider a class of stationary viscous Hamilton-Jacobi equations as

$$
\begin{cases}\lambda u-\operatorname{div}(A(x) \nabla u)=H(x, \nabla u) & \text { in } \Omega, \\ u=0 & \text { on } \partial \Omega\end{cases}
$$

where $\lambda \geq 0, A(x)$ is a bounded and uniformly elliptic matrix and $H(x, \xi)$ is convex in $\xi$ and grows at most like $|\xi|^{q}+f(x)$, with $1<q<2$ and $f \in$ $L^{N / q^{\prime}}(\Omega)$. Under such growth conditions solutions are in general unbounded, and there is not uniqueness of usual weak solutions. We prove that uniqueness holds in the restricted class of solutions satisfying a suitable energy-type estimate, i.e. $(1+|u|)^{\bar{q}-1} u \in H_{0}^{1}(\Omega)$, for a certain (optimal) exponent $\bar{q}$. This completes the recent results in [15], where the existence of at least one solution in this class has been proved.
\end{abstract}

Mathematics Subject Classification (2000): 35J60 (primary); 35R05, 35Dxx (secondary).

\section{Introduction}

In this paper we consider a class of elliptic equations in a bounded domain $\Omega \subset \mathbb{R}^{N}$, $N>2$

$$
\begin{cases}\lambda u-\operatorname{div}(A(x) \nabla u)=H(x, \nabla u) & \text { in } \Omega, \\ u=0 & \text { on } \partial \Omega\end{cases}
$$

where the function $H(x, \xi)$ is convex and superlinear with respect to $\xi$.

Equations of this type are sometimes referred to as stationary viscous HamiltonJacobi equations and appear in connection to stochastic optimal control problems. In that context, the convexity of $H$ is a natural assumption. The model example which we are going to treat is the following

$$
\begin{cases}\lambda u-\operatorname{div}(A(x) \nabla u)=\gamma|\nabla u|^{q}+f(x) & \text { in } \Omega, \\ u=0 & \text { on } \partial \Omega\end{cases}
$$

Received May 19, 2005; accepted in revised form March 13, 2006. 
where $q>1, \lambda \geq 0$ and $A(x)=\left(a_{i, j}(x)\right)$ is a matrix of $L^{\infty}(\Omega)$ functions $a_{i, j}(x)$ satisfying uniform ellipticity and boundedness conditions

$$
\alpha|\xi|^{2} \leq A(x) \xi \cdot \xi \leq \beta|\xi|^{2} \quad \forall \xi \in \mathbf{R}^{N}, \quad \text { a.e. } \quad x \in \Omega .
$$

Without loss of generality, we can take $\gamma>0$. We draw our attention to the "subcritical" case, namely $q<2$, and, more precisely, to the question of uniqueness of unbounded solutions.

Let us first recall that some regularity condition is needed on $f$ in order that problem (1.2) admits a solution. In the class of Lebesgue spaces, this condition amounts to ask that

$$
f \in L^{\frac{N}{q^{\prime}}}(\Omega),
$$

where $q^{\prime}$ is the conjugate exponent of $q$, i.e. $q^{\prime}=\frac{q}{q-1}$. When $q<2$, (1.4) implies that $f \in L^{m}(\Omega)$ with $m<\frac{N}{2}$, hence solutions are expected to be unbounded. Moreover since, by Sobolev embedding theorem, one has

$$
L^{\frac{N}{q^{\prime}}}(\Omega) \subset H^{-1}(\Omega) \quad \Longleftrightarrow \quad q \geq 1+\frac{2}{N},
$$

the value $q=1+\frac{2}{N}$ is a critical one. Indeed, the solutions belong to $H_{0}^{1}(\Omega)$ only if $q \geq 1+\frac{2}{N}$, when $q$ is below this value solutions are not only unbounded but have not even finite energy and should be defined in a suitable generalized sense.

The fact that (1.4) is a necessary condition for having solutions can be easily justified by a heuristic argument: if $A(x)=I$, i.e. in case of the Laplace operator, the Calderon-Zygmund regularity implies that

$$
-\Delta u \in L^{m}(\Omega) \Longrightarrow u \in W^{2, m}(\Omega) \Longrightarrow|\nabla u| \in L^{m^{*}}(\Omega),
$$

where $m^{*}$ is associated to $m$ through the Sobolev embedding, i.e., for $N>m$, $m^{*}=\frac{N m}{N-m}$. In order to be consistent with (1.2), this means that $f \in L^{m}(\Omega)$ and $|\nabla u| \in L^{q m}(\Omega)$ so that it should be $q m=m^{*}$, i.e. $m=\frac{N}{q^{\prime}}$. We refer the reader to $[1,16]$ for rigorous and sharper necessary conditions on $f$ in order to have weak solutions. It is important to recall that if $\lambda=0$ the data $f, \gamma, \alpha$ must also satisfy a size condition in order that a solution exists.

Pioneering results for such kind of equations were given by P.L. Lions ([17, 18]), mainly in case of Lipschitz solutions and including $q>2$. Existence results for the case $q=2$ can be found in several works, among which we recall the series of papers by L. Boccardo, F. Murat, J.P. Puel (see e.g. [8, 9]) and more recently, assuming $f$ in $L^{\frac{N}{2}}(\Omega)$, in $[13,10]$. 
Under assumption (1.4) with $1<q<2$, the existence of a solution for problems as (1.2) has been recently proved in [15] (see also [14]) if either $\lambda>0$ or $\lambda=0$ and a size condition is satisfied:

$$
\gamma^{\frac{1}{q-1}}\|f\|_{L^{\frac{N}{q^{\prime}}}(\Omega)}<\alpha^{q^{\prime}} C_{*},
$$

where $C_{*}$ only depends on $q$ and $N$.

In this paper we deal with the problem of uniqueness of solutions. Up to now, uniqueness results for problems like (1.2) have been proved in the Lipschitz case ([17]) and in [2,3] if either solutions are bounded or $q=2$. Note that these two cases share a common feature, which is that $f$ is required to be in $L^{m}(\Omega), m \geq \frac{N}{2}$ : for less summable $f$ as we consider, the approach of these previous papers seems not to work. Some results when $q \leq 1+\frac{2}{N}$ can be found in $[4,5]$.

When dealing with the question of uniqueness, one has to consider the following well-known counterexample (see also $[17,1]$ ) for $q>\frac{N}{N-1}$

$$
\begin{aligned}
& u(x)=C_{\alpha}\left(|x|^{\alpha}-1\right)\left(\alpha=-\frac{2-q}{q-1}, C_{\alpha}=\frac{(N+\alpha-2)^{\frac{1}{q-1}}}{|\alpha|}\right) \text { solves } \\
& \left\{\begin{array}{l}
-\Delta u=|\nabla u|^{q} \\
u \in W_{0}^{1, q}\left(B_{1}(0)\right) .
\end{array}\right.
\end{aligned}
$$

This shows that uniqueness does not hold in the class of weak solutions $u$ in $W_{0}^{1, q}(\Omega)$, and, if $q>1+\frac{2}{N}$, not even in $H_{0}^{1}(\Omega)$ (one can check that $u \in H_{0}^{1}(\Omega)$ in this case). It is then natural to look for a suitable class of solutions in which problem (1.2) is well-posed. A linearization argument would suggest that there is uniqueness in the class

$$
u \text { sol. of (1.2): }|\nabla u| \in L^{N(q-1)}(\Omega) .
$$

On the other hand, if $q>1+\frac{2}{N}$ (which gives $N(q-1)>2$ ), the existence of such kind of solutions can not be obtained unless the Calderon-Zygmund regularity theorem applies; thus, in order to deal with general (bounded measurable) coefficients $a_{i, j}$, this approach is not reasonable.

Our main purpose here is to prove the uniqueness of solutions of (1.2) in a regularity class which is consistent with the existence results available from [15]. In this latter paper it has been proved that a natural class of solutions for which both a priori estimates and existence hold is given through the extra energy condition

$$
u \text { sol. of (1.2): }(1+|u|)^{\bar{q}-1} u \in H_{0}^{1}(\Omega), \text { with } \bar{q}=\frac{(N-2)(q-1)}{2(2-q)} .
$$

We are going to prove that this regularity is precisely what is needed to select a unique solution, so that problem (1.2) is actually well-posed in this class.

Our main result concerns the case $q \geq 1+\frac{2}{N}$, which corresponds to $H_{0}^{1}(\Omega)$ solutions (see (1.5)). 
Theorem 1.1. Let $1+\frac{2}{N} \leq q<2$. Assume (1.3), (1.4) and that

(i) either $\lambda>0$

(ii) or $\lambda=0$ and (1.6) is satisfied.

Then problem (1.2) has at most one (distributional) solution $u$ such that $(1+$ $|u|)^{\bar{q}-1} u \in H_{0}^{1}(\Omega)$, with $\bar{q}=\frac{(N-2)(q-1)}{2(2-q)}$.

Note that the function $u$ in the counterexample (1.7) satisfies $(1+|u|)^{r-1} u \in$ $H_{0}^{1}(\Omega)$ for any $r<\bar{q}$ but not for $r=\bar{q}$, which proves the optimality of our result. Observe also that $\bar{q}$ tends to infinity as $q \rightarrow 2$, which is consistent with the case $f \in L^{\frac{N}{2}}(\Omega)$, for which existence and uniqueness have been proved (see [13, 3] respectively) in the class of solutions $u$ such that $\exp (\mu u)-1 \in H_{0}^{1}(\Omega)$ for a suitable constant $\mu$.

We leave to Section 2 the proof of Theorem 1.1, actually in a generalized version which includes problem (1.1) where $H$ is convex and satisfies similar growth conditions. Some extensions to Neumann boundary conditions as well as to the case of unbounded domains is also discussed.

In Section 3 we deal with the case $\frac{N}{N-1}<q<1+\frac{2}{N}$, which corresponds to $f \in L^{m}(\Omega)$ with $1<m<\frac{2 N}{N+2}$. A similar result as Theorem 1.1 is proved, but since, in this case, solutions do not belong to $H_{0}^{1}(\Omega)$, we use a slightly stronger formulation than the distributional one, namely uniqueness is proved for so-called renormalized solutions (still in the class (1.9)). This notion (see Definition 3.1), first introduced in [12] for transport equations, is now currently used in several different contexts when dealing with solutions of infinite energy.

Still in Section 3, we prove in fact a more general uniqueness result when $q$ is below the critical value $1+\frac{2}{N}$. Indeed, we will see that if $q \leq 1+\frac{2}{N}$ then the regularity (1.9) implies (1.8). This fact allows to prove uniqueness through a simpler linearization principle, which does not need any convexity argument and which can be applied to more general situations like, for instance, nonlinear operators (see Theorem 3.4). Note that the limiting value $q=1+\frac{2}{N}$ is also admitted here; actually, (1.9) and (1.8) coincide in that case with $u \in H_{0}^{1}(\Omega)$. On the other hand, as mentioned before, this argument was not possible for $q>1+\frac{2}{N}$ since (1.8) will no more be true in general.

Finally, some further remarks will be discussed at the end of Section 3, including the case $q<\frac{N}{N-1}$, where uniqueness holds simply in $W_{0}^{1, q}(\Omega)$.

\section{The case $q \geq 1+\frac{2}{N}$ : finite energy solutions}

We consider a natural generalization of (1.2), namely the following equation

$$
\begin{cases}\lambda u-\operatorname{div}(A(x) \nabla u)=H(x, \nabla u) & \text { in } \Omega, \\ u=0 & \text { on } \partial \Omega .\end{cases}
$$


We still assume that $\lambda \geq 0$, that $A(x)$ satisfies (1.3) and that $H(x, \xi)$ is a Carathéodory function satisfying, for a.e. $x \in \Omega$ and for every $\xi \in \mathbb{R}^{N}$,

$$
\xi \mapsto H(x, \xi) \quad \text { is convex, for a.e. } x \in \Omega,
$$

and the growth condition

$\exists q \in\left(\frac{N}{N-1}, 2\right): \quad|H(x, \xi)| \leq \gamma|\xi|^{q}+f(x), \quad \gamma>0, \quad f(x) \in L^{\frac{N}{q^{\prime}}}(\Omega)$.

Note that this assumptions include the possibility that the equation contains transport terms; indeed, a possible choice for $H$ is

$$
H(x, \nabla u)=b(x) \cdot \nabla u+\gamma(x)|\nabla u|^{p}+f(x)
$$

where $b \in\left[L^{N}(\Omega)\right]^{N}, \gamma \in L^{r}(\Omega)$ and $f \in L^{\frac{N r(p-1)}{p r-N}}(\Omega)$, with $r \in(N,+\infty]$ and $\frac{N(r-1)}{r(N-1)}<p<2-\frac{N}{r}$.

In virtue of (2.3) and (1.5), assuming $q \geq 1+\frac{2}{N}$ corresponds to having data in $H^{-1}(\Omega)$, so that we can reasonably talk of $H_{0}^{1}(\Omega)$ weak solutions.

Definition 2.1. We say that $u \in H_{0}^{1}(\Omega)$ is a weak subsolution of $(2.1)$ if $H(x, \nabla u) \in$ $L^{1}(\Omega)$ and

$$
\begin{gathered}
\lambda \int_{\Omega} u \xi d x+\int_{\Omega} A(x) \nabla u \nabla \xi d x \leq \int_{\Omega} H(x, \nabla u) \xi d x \\
\forall \xi \in H_{0}^{1}(\Omega) \cap L^{\infty}(\Omega), \quad \xi \geq 0 .
\end{gathered}
$$

A supersolution of (2.1) is defined if the opposite inequality holds. A function $u$ being both a subsolution and a supersolution is said to be a weak solution of (2.1).

Our proof of the comparison principle for sub and supersolutions of (2.1) relies on two basic ideas: the first one is that if

$$
\left\{\begin{array}{l}
-\operatorname{div}(A(x) \nabla w) \leq C|\nabla w|^{q} \\
\left(w^{+}\right)^{\bar{q}} \in H_{0}^{1}(\Omega),
\end{array}\right.
$$

then $w \leq 0$; in other words, the homogeneous problem has only the trivial solution in this regularity class. Secondly, we aim at applying inequality (2.5) to (a small perturbation of) the difference of two solutions $u-v$. In order to do that, we use a convexity argument, which gives account of assumption (2.2).

A further technical tool will be required in order to justify some regularity claimed on $u-v$ : here we apply a truncation argument. To this purpose, we introduce the following truncation function

$$
T_{n}(s)=\int_{0}^{s} \theta_{n}(\xi) d \xi, \quad \theta_{n}(\xi)= \begin{cases}1 & \text { if }|\xi|<n \\ \frac{2 n-|\xi|}{n} & \text { if } n<|\xi|<2 n \\ 0 & \text { if }|\xi|>2 n\end{cases}
$$

and we start by giving a sort of renormalization principle for the "truncated" equation. 
Lemma 2.2. Let $u \in H_{0}^{1}(\Omega)$ be a weak subsolution of (2.1). Then $u$ satisfies, for any nonnegative $\xi \in H_{0}^{1}(\Omega) \cap L^{\infty}(\Omega)$ and for every $n$

$\lambda \int_{\Omega} T_{n}(u) \xi d x+\int_{\Omega} A(x) \nabla T_{n}(u) \nabla \xi d x \leq \int_{\Omega} H\left(x, \nabla T_{n}(u)\right) \xi d x+\left\langle I_{n}^{u}, \xi\right\rangle$,

where $I_{n}^{u} \in L^{1}(\Omega)$ and is defined as follows:

$$
\begin{aligned}
\left\langle I_{n}^{u}, \xi\right\rangle= & \frac{1}{n} \int_{\{n<u<2 n\}} A(x) \nabla u \nabla u \xi d x-\frac{1}{n} \int_{\{-2 n<u<-n\}} A(x) \nabla u \nabla u \xi d x \\
& +\lambda \int_{\Omega}\left(T_{n}(u)-u \theta_{n}(u)\right) \xi d x \\
& +\int_{\Omega}\left(H(x, \nabla u) \theta_{n}(u)-H\left(x, \nabla T_{n}(u)\right)\right) \xi d x
\end{aligned}
$$

If moreover $|u|^{\bar{q}-1} u \in H_{0}^{1}(\Omega)$, where $\bar{q}=\frac{(N-2)(q-1)}{2(2-q)}$, we have

$$
\lim _{n \rightarrow+\infty} n^{2 \bar{q}-1}\left\|I_{n}^{u}\right\|_{L^{1}(\Omega)}=0
$$

Proof. Let $\xi \in H_{0}^{1}(\Omega) \cap L^{\infty}(\Omega), \xi \geq 0$, and let $n>0$. Choosing in (2.4) the test function $\theta_{n}(u) \xi$ we obtain

$$
\begin{gathered}
\lambda \int_{\Omega} u \theta_{n}(u) \xi d x+\int_{\Omega} A(x) \nabla u \nabla \xi \theta_{n}(u) d x \leq \int_{\Omega} H(x, \nabla u) \theta_{n}(u) \xi d x \\
\quad+\frac{1}{n} \int_{\{n<u<2 n\}} A(x) \nabla u \nabla u \xi d x-\frac{1}{n} \int_{\{-2 n<u<-n\}} A(x) \nabla u \nabla u \xi d x .
\end{gathered}
$$

Recalling that $\theta_{n}(u)=T_{n}^{\prime}(u)$ and defining $I_{n}^{u}$ as in (2.8) we have obtained (2.7). Now let $u$ be such that $|u|^{\bar{q}-1} u \in H_{0}^{1}(\Omega)$, where $\bar{q}=\frac{(N-2)(q-1)}{2(2-q)}$. We have, by definition of $I_{n}^{u}$

$$
\begin{aligned}
& n^{2 \bar{q}-1}\left\|I_{n}^{u}\right\|_{L^{1}(\Omega)} \\
\leq & n^{2 \bar{q}-1}\left[\frac{1}{n} \int_{\{n<u<2 n\}} A(x) \nabla u \nabla u d x+\frac{1}{n} \int_{\{-2 n<u<-n\}} A(x) \nabla u \nabla u d x\right] \\
& +\lambda n^{2 \bar{q}-1} \int_{\Omega}\left|T_{n}(u)-u \theta_{n}(u)\right| d x+n^{2 \bar{q}-1} \int_{\Omega}\left|H(x, \nabla u) \theta_{n}(u)-H\left(x, \nabla T_{n}(u)\right)\right| d x
\end{aligned}
$$


Observe that

$$
\begin{aligned}
& n^{2 \bar{q}-1} \frac{1}{n} \int_{\{n<u<2 n\}} A(x) \nabla u \nabla u d x \leq \int_{\{n<u<2 n\}} A(x) \nabla u \nabla u|u|^{2 \bar{q}-2} d x \\
& \leq\left.\left.\frac{\beta}{\bar{q}^{2}} \int_{\{n<u<2 n\}}|\nabla| u\right|^{\bar{q}}\right|^{2} d x \stackrel{n \rightarrow+\infty}{\rightarrow} 0,
\end{aligned}
$$

and similarly

$$
n^{2 \bar{q}-2} \int_{\{-2 n<u<-n\}} A(x) \nabla u \nabla u d x \stackrel{n \rightarrow+\infty}{\rightarrow} 0 .
$$

We also have, using Young's inequality, and by definition of $\bar{q}$,

$$
\begin{aligned}
|\nabla u|^{q}|u|^{2 \bar{q}-1} & \leq \frac{q}{2}|\nabla u|^{2}|u|^{2(\bar{q}-1)}+\frac{2-q}{2}|u|^{2 \bar{q}+\frac{2(q-1)}{2-q}} \\
& =\frac{q}{2 \bar{q}^{2}}\left|\nabla\left(|u|^{\bar{q}-1} u\right)\right|^{2}+\frac{2-q}{2}\left(|u|^{\bar{q}}\right)^{2^{*}} .
\end{aligned}
$$

Since $|u|^{\bar{q}-1} u \in H_{0}^{1}(\Omega)$, using Sobolev embedding, we conclude that

$$
|\nabla u|^{q}|u|^{2 \bar{q}-1} \in L^{1}(\Omega) .
$$

Similarly, since $(2 \bar{q}-1)\left(\frac{N}{q^{\prime}}\right)^{\prime}=2^{*} \bar{q}$ we have that $|u|^{2 \bar{q}-1} \in L^{\left(\frac{N}{q^{\prime}}\right)^{\prime}}(\Omega)$; since $f \in L^{\frac{N}{q^{\prime}}}(\Omega)$ we deduce that

$$
f|u|^{2 \bar{q}-1} \in L^{1}(\Omega) .
$$

Now we have, by definition of $\theta_{n}$ and $T_{n}$,

$$
\left|H(x, \nabla u) \theta_{n}(u)-H\left(x, \nabla T_{n}(u)\right)\right| \leq\left[|H(x, \nabla u)|+\left|H\left(x, \nabla T_{n}(u)\right)\right|\right] \chi_{\{|u|>n\}},
$$

hence, using the growth assumption (2.3),

$$
n^{2 \bar{q}-1}\left|H(x, \nabla u) \theta_{n}(u)-H\left(x, \nabla T_{n}(u)\right)\right| \leq 2\left(\gamma|\nabla u|^{q}+f(x)\right)|u|^{2 \bar{q}-1} \chi_{\{|u|>n\}} .
$$

Thanks to (2.14)-(2.15) we conclude

$$
n^{2 \bar{q}-1} \int_{\Omega}\left|H(x, \nabla u) \theta_{n}(u)-H\left(x, \nabla T_{n}(u)\right)\right| d x \stackrel{n \rightarrow+\infty}{\longrightarrow} 0 .
$$

Finally, since $u \in L^{2^{*} \bar{q}}(\Omega)$,

$$
n^{2 \bar{q}-1} \int_{\Omega}\left|T_{n}(u)-u \theta_{n}(u)\right| d x \leq 2 \int_{\Omega}|u|^{2 \bar{q}} \chi_{\{|u|>n\}} d x \stackrel{n \rightarrow+\infty}{\longrightarrow} 0 .
$$

From (2.11), (2.12), (2.13), (2.16), (2.17) we get (2.9). 
Remark 2.3. If $u \in H_{0}^{1}(\Omega)$ is a supersolution of (2.1) then (2.7) holds with the opposite sign, and (2.9) still holds true. In particular, if $u$ is a weak solution of (2.1), then

$$
\lambda \int_{\Omega} T_{n}(u) \xi d x+\int_{\Omega} A(x) \nabla T_{n}(u) \nabla \xi d x=\int_{\Omega} H\left(x, \nabla T_{n}(u)\right) \xi d x+\left\langle I_{n}^{u}, \xi\right\rangle,
$$

with $n^{2 \bar{q}-1}\left\|I_{n}^{u}\right\|_{L^{1}(\Omega)} \rightarrow 0$ provided $|u|^{\bar{q}-1} u \in H_{0}^{1}(\Omega)$.

We come to the main comparison result.

Theorem 2.4. Assume (1.3), (2.2), (2.3) with $q \geq 1+\frac{2}{N}$. Let $\lambda>0$. If $u$ and $v$ are respectively a subsolution and a supersolution of (2.1) such that $(1+|u|)^{\bar{q}-1} u \in$ $H_{0}^{1}(\Omega)$ and $(1+|v|)^{\bar{q}-1} v \in H_{0}^{1}(\Omega)$, then we have $u \leq v$ in $\Omega$.

In particular, problem (2.1) has at most one weak solution u such that $(1+$ $|u|)^{\bar{q}-1} u \in H_{0}^{1}(\Omega)$.

Proof. From Lemma 2.2 we obtain that

$\lambda \int_{\Omega} T_{n}(u) \xi d x+\int_{\Omega} A(x) \nabla T_{n}(u) \nabla \xi d x \leq \int_{\Omega} H\left(x, \nabla T_{n}(u)\right) \xi d x+\left\langle I_{n}^{u}, \xi\right\rangle$,

and

$\lambda \int_{\Omega} T_{n}(v) \xi d x+\int_{\Omega} A(x) \nabla T_{n}(v) \nabla \xi d x \geq \int_{\Omega} H\left(x, \nabla T_{n}(v)\right) \xi d x+\left\langle I_{n}^{v}, \xi\right\rangle$,

for every $\xi \in H_{0}^{1}(\Omega) \cap L^{\infty}(\Omega), \xi \geq 0$.

Let now $\varepsilon \in(0,1)$ be fixed. Subtracting (2.18) and (2.19), we obtain

$$
\begin{aligned}
& \lambda \int_{\Omega}\left[T_{n}(u)-(1-\varepsilon) T_{n}(v)\right] \xi d x+\int_{\Omega} A(x) \nabla\left(T_{n}(u)-(1-\varepsilon) T_{n}(v)\right) \nabla \xi d x \\
& \quad \leq \int_{\Omega}\left[H\left(x, \nabla T_{n}(u)\right)-(1-\varepsilon) H\left(x, \nabla T_{n}(v)\right)\right] \xi d x+\left\langle I_{n}^{u}, \xi\right\rangle-(1-\varepsilon)\left\langle I_{n}^{v}, \xi\right\rangle .
\end{aligned}
$$

Now we use the convexity assumption on $H$, which gives

$$
H(x, p) \leq(1-\varepsilon) H(x, \eta)+\varepsilon H\left(x, \frac{p-(1-\varepsilon) \eta}{\varepsilon}\right), \quad \forall p, \eta \in \mathbf{R}^{N} .
$$

With $p=\nabla T_{n}(u), \eta=\nabla T_{n}(v)$ we obtain

$$
H\left(x, \nabla T_{n}(u)\right)-(1-\varepsilon) H\left(x, \nabla T_{n}(v)\right) \leq \varepsilon H\left(x, \frac{\nabla T_{n}(u)-(1-\varepsilon) \nabla T_{n}(v)}{\varepsilon}\right),
$$

hence we have

$$
\begin{aligned}
& \lambda \int_{\Omega}\left[T_{n}(u)-(1-\varepsilon) T_{n}(v)\right] \xi d x+\int_{\Omega} A(x) \nabla\left[T_{n}(u)-(1-\varepsilon) T_{n}(v)\right] \nabla \xi d x \\
& \leq \varepsilon \int_{\Omega} H\left(x, \frac{\nabla\left[T_{n}(u)-(1-\varepsilon) T_{n}(v)\right]}{\varepsilon}\right) \xi d x+\left\langle I_{n}^{u}, \xi\right\rangle-(1-\varepsilon)\left\langle I_{n}^{v}, \xi\right\rangle .
\end{aligned}
$$


We define now

$$
w_{n}=T_{n}(u)-(1-\varepsilon) T_{n}(v)-\varepsilon \varphi,
$$

where $\varphi$ is a positive function that belongs to $H_{0}^{1}(\Omega)$ and will be chosen later. From (2.20) we obtain

$$
\begin{gathered}
\lambda \int_{\Omega} w_{n} \xi d x+\int_{\Omega} A(x) \nabla w_{n} \nabla \xi d x \leq \varepsilon \int_{\Omega} H\left(x, \frac{\nabla w_{n}}{\varepsilon}+\nabla \varphi\right) \xi d x \\
-\varepsilon\left[\int_{\Omega} \lambda \varphi \xi+A(x) \nabla \varphi \nabla \xi d x\right]+\left\langle I_{n}^{u}, \xi\right\rangle-(1-\varepsilon)\left\langle I_{n}^{v}, \xi\right\rangle .
\end{gathered}
$$

Using assumption (2.3) we have

$$
\begin{aligned}
& H\left(x, \frac{\nabla w_{n}}{\varepsilon}+\nabla \varphi\right) \leq \gamma\left|\frac{\nabla w_{n}}{\varepsilon}+\nabla \varphi\right|^{q}+f(x) \\
& \quad \leq(\gamma+\delta)|\nabla \varphi|^{q}+C_{\delta}\left|\frac{\nabla w_{n}}{\varepsilon}\right|^{q}+f(x),
\end{aligned}
$$

where $\delta$ is any positive constant.

Using (2.23) in (2.22) we obtain

$$
\begin{aligned}
& \lambda \int_{\Omega} w_{n} \xi d x+\int_{\Omega} A(x) \nabla w_{n} \nabla \xi d x \leq \frac{C_{\delta}}{\varepsilon^{q-1}} \int_{\Omega}\left|\nabla w_{n}\right|^{q} \xi d x \\
& \quad-\varepsilon\left[\int_{\Omega}\left\{\lambda \varphi \xi+A(x) \nabla \varphi \nabla \xi-(\gamma+\delta)|\nabla \varphi|^{q} \xi-f(x) \xi\right\} d x\right] \\
& \quad+\left\langle I_{n}^{u}, \xi\right\rangle-(1-\varepsilon)\left\langle I_{n}^{v}, \xi\right\rangle .
\end{aligned}
$$

We choose now $\varphi$ as a solution of

$$
\left\{\begin{array}{l}
\lambda \varphi-\operatorname{div}(A(x) \nabla \varphi)=(\gamma+\delta)|\nabla \varphi|^{q}+f(x) \quad \text { in } \Omega \\
(1+|\varphi|)^{\bar{q}-1} \varphi \in H_{0}^{1}(\Omega)
\end{array}\right.
$$

The existence of such a function $\varphi$ is proved in [14]. Moreover, we have that $\varphi \geq 0$ (since $f \geq 0$ from (2.3)). Thanks to (2.25) we obtain from (2.24)

$$
\begin{aligned}
\lambda \int_{\Omega} w_{n} \xi d x+\int_{\Omega} A(x) \nabla w_{n} \nabla \xi d x \leq & \frac{C_{\delta}}{\varepsilon^{q-1}} \int_{\Omega}\left|\nabla w_{n}\right|^{q} \xi d x \\
& +\left\langle I_{n}^{u}, \xi\right\rangle-(1-\varepsilon)\left\langle I_{n}^{v}, \xi\right\rangle .
\end{aligned}
$$

For $l>0$, we choose in (2.26) $\xi=\xi_{n}$ defined as

$$
\xi_{n}=\left[\left(w_{n}-l\right)^{+}\right]^{2 \bar{q}-1} .
$$


Note that $\xi_{n}$ is a positive function, and it belongs to $H_{0}^{1}(\Omega) \cap L^{\infty}(\Omega)$. Moreover, by the definition of $w_{n}$ in (2.21), we have

$$
\left\|\xi_{n}\right\|_{L^{\infty}(\Omega)} \leq(2 n)^{2 \bar{q}-1},
$$

so that we can apply Lemma 2.2 for $u$ and $v$ and get

$$
\begin{aligned}
& \left|\left\langle I_{n}^{u}, \xi_{n}\right\rangle\right| \leq(2 n)^{2 \bar{q}-1}\left\|I_{n}^{u}\right\|_{L^{1}(\Omega)} \stackrel{n \rightarrow+\infty}{\longrightarrow} 0 \\
& \left|\left\langle I_{n}^{v}, \xi_{n}\right\rangle\right| \leq(2 n)^{2 \bar{q}-1}\left\|I_{n}^{v}\right\|_{L^{1}(\Omega)} \stackrel{n \rightarrow+\infty}{\longrightarrow} 0 .
\end{aligned}
$$

Thus (2.26) implies

$$
\begin{aligned}
& \lambda \int_{\Omega} w_{n}\left[\left(w_{n}-l\right)^{+}\right]^{2 \bar{q}-1} d x+(2 \bar{q}-1) \int_{\Omega} A(x) \nabla w_{n} \nabla w_{n}\left[\left(w_{n}-l\right)^{+}\right]^{2 \bar{q}-2} d x \\
& \quad \leq \frac{C_{\delta}}{\varepsilon^{q-1}} \int_{\Omega}\left|\nabla w_{n}\right|^{q}\left[\left(w_{n}-l\right)^{+}\right]^{2 \bar{q}-1} d x+o(1)_{n},
\end{aligned}
$$

where $o(1)_{n}$ goes to zero as $n$ tends to infinity. Neglecting the zero order term which is positive, and using that $A(x) \geq \alpha I$, we have

$$
\begin{aligned}
& \alpha(2 \bar{q}-1) \int_{\Omega}\left|\nabla w_{n}\right|^{2}\left[\left(w_{n}-l\right)^{+}\right]^{2 \bar{q}-2} d x \\
& \leq \frac{C_{\delta}}{\varepsilon^{q-1}} \int_{\Omega}\left|\nabla w_{n}\right|^{q}\left[\left(w_{n}-l\right)^{+}\right]^{2 \bar{q}-1} d x+o(1)_{n} .
\end{aligned}
$$

Young's inequality implies

$$
\begin{aligned}
\frac{C_{\delta}}{\varepsilon^{q-1}} \int_{\Omega}\left|\nabla w_{n}\right|^{q}\left[\left(w_{n}-l\right)^{+}\right]^{2 \bar{q}-1} d x \leq & \frac{\alpha}{2}(2 \bar{q}-1) \int_{\Omega}\left|\nabla w_{n}\right|^{2}\left[\left(w_{n}-l\right)^{+}\right]^{2 \bar{q}-2} d x \\
& +C_{\varepsilon, \delta} \int_{\Omega}\left[\left(w_{n}-l\right)^{+}\right]^{2 \bar{q}+\frac{2(q-1)}{2-q}} d x,
\end{aligned}
$$

hence, using that $2 \bar{q}+\frac{2(q-1)}{2-q}=\bar{q} 2^{*}$ we get

$$
\frac{\alpha}{2}(2 \bar{q}-1) \int_{\Omega}\left|\nabla w_{n}\right|^{2}\left[\left(w_{n}-l\right)^{+}\right]^{2 \bar{q}-2} d x \leq C_{\varepsilon, \delta} \int_{\Omega}\left[\left(w_{n}-l\right)^{+}\right]^{\bar{q} 2^{*}} d x+o(1)_{n} .
$$

Using Sobolev inequality in the left hand side we obtain

$$
C\left(\int_{\Omega}\left[\left(w_{n}-l\right)^{+}\right]^{\bar{q} 2^{*}} d x\right)^{1-\frac{2}{N}} \leq C_{\varepsilon, \delta} \int_{\Omega}\left[\left(w_{n}-l\right)^{+}\right]^{\bar{q} 2^{*}} d x+o(1)_{n} .
$$

We let now $n$ tend to infinity; since $u, v$ and $\varphi$ all belong to $L^{\bar{q} 2^{*}}(\Omega)$, we have that $w_{n} \rightarrow w:=u-(1-\varepsilon) v-\varepsilon \varphi \quad$ strongly in $L^{\bar{q} 2^{*}}(\Omega)$ as $n$ tends to infinity, 
hence we get

$$
C\left(\int_{\Omega}\left[(w-l)^{+}\right]^{\bar{q} 2^{*}} d x\right)^{1-\frac{2}{N}} \leq C_{\varepsilon, \delta} \int_{\Omega}\left[(w-l)^{+}\right]^{\bar{q} 2^{*}} d x .
$$

Since $1-\frac{2}{N}<1$, last inequality implies that $w \leq 0$; indeed, if $\sup w>0$ (even possibly infinite), one gets a contradiction by letting $l$ converge to sup $w$ and using that $\left[(w-l)^{+}\right]^{\bar{q} 2^{*}}$ would tend to zero in $L^{1}(\Omega)$.

The conclusion is that $w \leq 0$, i.e.

$$
u \leq(1-\varepsilon) v+\varepsilon \varphi,
$$

and, letting $\varepsilon \rightarrow 0, u \leq v$ in $\Omega$.

Let us now deal with the case $\lambda=0$. Then, the same proof can be applied provided there exists a solution of

$$
\begin{cases}-\operatorname{div}(A(x) \nabla \varphi)=(\gamma+\delta)|\nabla \varphi|^{q}+f(x) & \text { in } \Omega, \\ \varphi=0 & \text { on } \partial \Omega,\end{cases}
$$

for some $\delta>0$. This requires a further assumption, which is a sort of size condition on the data.

Indeed, it is known from [14] that there exists a constant $C_{*}$, only depending on $q$ and $N$, such that if

$$
b^{\frac{1}{q-1}}\|f\|_{L^{\frac{N}{q^{\prime}}}(\Omega)}<\alpha^{q^{\prime}} C_{*}
$$

then the problem

$$
\begin{cases}-\operatorname{div}(A(x) \nabla z)=b|\nabla z|^{q}+f(x) & \text { in } \Omega \\ z=0 & \text { on } \partial \Omega\end{cases}
$$

admits a solution $z$ such that $(1+|z|)^{\bar{q}-1} z \in H_{0}^{1}(\Omega)$. In particular, if we fix $\alpha$ (the coercivity constant of $A(x))$ and $f$, the set

$$
\mathcal{B}_{f}:=\left\{b>0: \text { problem }(2.30) \text { has a solution } z:(1+|z|)^{\bar{q}-1} z \in H_{0}^{1}(\Omega)\right\}
$$

is non empty. Indeed, it is not difficult to see that $\mathcal{B}_{f}$ is even an interval. In order to assure that (2.28) has a solution for a certain $\delta>0$, we are then led to assume that (2.3) holds with $\gamma<\sup \mathcal{B}_{f}$.

Theorem 2.5. Let $\lambda=0$. Assume (1.3), (2.2) and (2.3) with $q \geq 1+\frac{2}{N}$ and $\gamma<\sup \mathcal{B}_{f}$, which is defined above. If $u$ and $v$ are respectively a subsolution and $a$ supersolution of (2.1) such that $(1+|u|)^{\bar{q}-1} u \in H_{0}^{1}(\Omega)$ and $(1+|v|)^{\bar{q}-1} v \in H_{0}^{1}(\Omega)$, then we have $u \leq v$ in $\Omega$. $H_{0}^{1}(\Omega)$. 
The result of Theorem 2.5 may also be rephrased more explicitly in terms of a size condition on the norm of $f$. Indeed, let $C_{*}$ be the maximal possible choice in (2.29), i.e.

$$
\begin{gathered}
C_{*}=\sup \left\{C>0: \text { if } \alpha^{-q^{\prime}} b^{\frac{1}{q-1}}\|f\|_{L^{\frac{N}{q^{\prime}}}(\Omega)}<C\right. \text { then problem (2.30) } \\
\text { has a solution } \left.z:(1+|z|)^{\bar{q}-1} z \in H_{0}^{1}(\Omega)\right\} .
\end{gathered}
$$

Then we have:

Corollary 2.6. Let $\lambda=0$. Assume (1.3), (2.2) and (2.3) with $q \geq 1+\frac{2}{N}$ and

$$
\alpha^{-q^{\prime}} \gamma^{\frac{1}{q-1}}\|f\|_{L^{\frac{N}{q^{\prime}}(\Omega)}}<C_{*} .
$$

Then problem (2.1) has at most one solution $u$ such that $(1+|u|)^{\bar{q}-1} u \in H_{0}^{1}(\Omega)$.

Remark 2.7. Applying Theorem 2.4 and Corollary 2.6 to the model problem

$$
\begin{cases}\lambda u-\operatorname{div}(A(x) \nabla u)=\gamma|\nabla u|^{q}+f(x) & \text { in } \Omega, \\ u=0 & \text { on } \partial \Omega\end{cases}
$$

we obtain the results stated in Theorem 1.1. Observe that if $\gamma>0$ and $f \in L^{\frac{N}{q^{\prime}}}(\Omega)$, one can easily prove that any weak solution satisfies $\left(u^{-}\right)^{\bar{q}} \in H_{0}^{1}(\Omega)$; in particular, in that case uniqueness holds in the class of solutions $u \in H_{0}^{1}(\Omega)$ such that $\left(u^{+}\right)^{\bar{q}} \in$ $H_{0}^{1}(\Omega)$. Clearly, when $\gamma$ is negative we should apply the result to the equation satisfied by $-u$.

Remark 2.8. When considering the case $\lambda=0$, a more careful look at the proof of Theorem 2.4 shows that in the inequality (2.23) one could replace $f$ with $\tilde{f}=$ $\sup \left(H(x, \xi)-\gamma|\xi|^{q}\right)^{+}$. The size condition of Theorem 2.5 and Corollary 2.6 would $\xi$

then concern $\tilde{f}$ instead of $f$. If one looks at the model problem (2.32), this simply means that if $\lambda=0$ and $\gamma>0$, the required size condition only concerns $f^{+}$, as it is expected.

Remark 2.9. When $q \rightarrow 2$, the exponent $\bar{q} \rightarrow+\infty$. In fact, if $q=2$ uniqueness for problems like (2.32) holds in the class of solutions $u \in H_{0}^{1}(\Omega)$ such that $e^{\mu u}-$ $1 \in H_{0}^{1}(\Omega)$ for some suitable $\mu>0$. This result is proved (in a more general framework) in [3]: the idea is to use the change of unknown function $v=e^{\gamma u}-1$, so that the standard choice is to take $\mu=\gamma$ and to prove uniqueness when $v \in$ $H_{0}^{1}(\Omega)$. Otherwise one could take $\mu=n \gamma$ for some $n>1$; in that case one proves uniqueness for solutions such that $|v|^{n-1} v \in H_{0}^{1}(\Omega)$. However, we point out that this latter choice requires to apply to the equation of $v$ a similar truncation argument as in Lemma 2.2. 


\subsection{Comments and extensions}

1. Data in $W^{-1, r}$

The results of this section still hold if the right hand side in (2.1) is replaced by $H(x, \nabla u)+\operatorname{div}(g(x))$ with $g(x) \in L^{N(q-1)}(\Omega)$.

2. Neumann boundary conditions

Our method easily extends to prove a comparison principle for the homogeneous Neumann problem which can be written in a strong form as

$$
\begin{cases}\lambda u-\operatorname{div}(A(x) \nabla u)=H(x, \nabla u) & \text { in } \Omega \\ A(x) \nabla u \cdot v(x)=0 & \text { on } \partial \Omega\end{cases}
$$

where $v(x)$ is the outward, unit normal vector to $\partial \Omega$ at $x$. Of course, we use the classical weak formulation which says that $u \in H^{1}(\Omega)$ is a weak solution of (2.33) if

$\lambda \int_{\Omega} u \varphi d x+\int_{\Omega} A(x) \nabla u \nabla \varphi d x=\int_{\Omega} H(x, \nabla u) \varphi d x \quad \forall \varphi \in H^{1}(\Omega) \cap L^{\infty}(\Omega)$.

Then one has:

Theorem 2.10. Assume (1.3), (2.2), (2.3) and that $\lambda>0$. Let $q \geq 1+\frac{2}{N}$. If $u$ and $v$ are respectively $a$ subsolution and a supersolution of (2.33) such that $(1+|u|)^{\bar{q}-1} u \in H^{1}(\Omega)$ and $(1+|v|)^{\bar{q}-1} v \in H^{1}(\Omega)$, then we have $u \leq v$ in $\Omega$. In particular, problem (2.33) has at most one weak solution u such that $(1+$ $|u|)^{\bar{q}-1} u \in H^{1}(\Omega)$.

Proof. The proof follows the same steps as for Theorem 2.4. Note that Lemma 2.2 is still true without any modification. Then one defines $\varphi$ as a solution of

$$
\begin{cases}\lambda \varphi-\operatorname{div}(A(x) \nabla \varphi)=(\gamma+\delta)|\nabla \varphi|^{q}+f(x) & \text { in } \Omega \\ A(x) \nabla \varphi \cdot v(x)=0 & \text { on } \partial \Omega\end{cases}
$$

and, setting $w_{n}=T_{n}(u)-(1-\varepsilon) T_{n}(v)-\varepsilon \varphi$, one obtains

$$
\begin{gathered}
\lambda \int_{\Omega} w_{n}\left[\left(w_{n}-l\right)^{+}\right]^{2 \bar{q}-1} d x+\frac{\alpha}{2}(2 \bar{q}-1) \int_{\Omega}\left|\nabla w_{n}\right|^{2}\left[\left(w_{n}-l\right)^{+}\right]^{2 \bar{q}-2} d x \\
\leq C_{\varepsilon, \delta} \int_{\Omega}\left[\left(w_{n}-l\right)^{+}\right]^{\bar{q} 2^{*}} d x+o(1)_{n} .
\end{gathered}
$$

Since $\lambda>0$ one deduces

$$
\left\|\left[\left(w_{n}-l\right)^{+}\right]^{\bar{q}}\right\|_{H^{1}(\Omega)} \leq \tilde{C}_{\varepsilon, \delta} \int_{\Omega}\left[\left(w_{n}-l\right)^{+}\right]^{\bar{q} 2^{*}} d x+o(1)_{n} .
$$

Using now Sobolev inequality one concludes as in the Dirichlet case. We only need to require here that $\Omega$ has enough regularity so that the Sobolev inequality holds. 


\section{Unbounded domains}

A slight refinement of our proof gives a similar result in case of unbounded domains. To be more precise, let $\Omega$ be a general domain, not necessarily bounded. Let still $q \geq 1+\frac{2}{N}$ and set $\bar{q}=\frac{(N-2)(q-1)}{2(2-q)}$. We denote by $D_{0}^{1,2}(\Omega)$ the space obtained by completion of functions $\varphi \in C_{c}^{\infty}(\Omega)$ with respect to the norm $\|\nabla \varphi\|_{L^{2}(\Omega)}$. It is well known that $D_{0}^{1,2}(\Omega)$ is a Banach space which, due to Sobolev inequality, is continuously embedded into $L^{2^{*}}(\Omega)$, namely

$$
\|v\|_{L^{2^{*}}(\Omega)} \leq S_{N}\|\nabla v\|_{L^{2}(\Omega)}=S_{N}\|v\|_{D_{0}^{1,2}(\Omega)} \quad \forall v \in D_{0}^{1,2}(\Omega),
$$

where $S_{N}$, the Sobolev constant, does not depend on $\Omega$, but only on the dimension $N$.

By a solution of (2.1) we mean a function $u$ such that

$$
u \psi \in H_{0}^{1}(\Omega) \quad \forall \psi \in C_{c}^{\infty}\left(\mathbf{R}^{N}\right), \quad|u|^{\bar{q}-1} u \in D_{0}^{1,2}(\Omega)
$$

and

$$
\lambda \int_{\Omega} u \xi d x+\int_{\Omega} A(x) \nabla u \nabla \xi d x=\int_{\Omega} H(x, \nabla u) \xi d x \quad \forall \xi \in C_{c}^{\infty}(\Omega) .
$$

Note that condition (2.34) gives a meaning to the Dirichlet condition on $\partial \Omega$; roughly speaking, one has (in a weak sense) $u=0$ on $\partial \Omega \cap B_{R}$ for any ball $B_{R}$. Under assumptions (1.3), (2.2)-(2.3), the existence of a solution of (2.1) in the sense of (2.34)-(2.35) has been proved in [15]. It was also pointed out that, due to the regularity of $|u|^{\bar{q}-1} u$, one can use in (2.35) any test function $\xi$ of the form $S(u)$, where $S(0)=0$ and $\left|S^{\prime}(t)\right| \leq|t|^{2 \bar{q}-2}$. This is achieved by choosing $\xi=S(u) \zeta\left(\frac{|x|}{n}\right)$, where $\zeta \in C_{c}^{\infty}\left(B_{2}\right), \zeta \equiv 1$ on $B_{1}$, and letting $n$ go to infinity, which is allowed thanks to (2.34) and (2.2)-(2.3).

Theorem 2.11. Assume (1.3), (2.2), (2.3) with $q \geq 1+\frac{2}{N}$, and that

(i) either $\lambda>0$

(ii) or $\lambda=0$ and (2.31) holds true.

Then there exists at most one solution $u$ of (2.1) in the sense of (2.34)-(2.35).

Proof. Note that Lemma 2.2 still holds true, i.e. (2.7) holds for any $\xi \in C_{c}^{\infty}\left(\mathbf{R}^{N}\right)$, and estimate (2.9) is still valid, since it only depends on the fact that $|u|^{\bar{q}-1} u \in$ $D_{0}^{1,2}(\Omega)$. We proceed then as in Theorem 2.4: let $\varphi$ be a solution (whose existence is proved in [15]) of the auxiliary problem

$$
\begin{cases}\lambda \varphi-\operatorname{div}(A(x) \nabla \varphi)=(\gamma+\delta)|\nabla \varphi|^{q}+f(x) & \text { in } \Omega, \\ |\varphi|^{\bar{q}-1} \varphi \in D_{0}^{1,2}(\Omega), \quad \varphi \psi \in H_{0}^{1}(\Omega) \text { for any } \psi \in C_{c}^{\infty}\left(\mathbf{R}^{N}\right) . & \end{cases}
$$

Defining $w_{n}=T_{n}(u)-(1-\varepsilon) T_{n}(v)-\varepsilon \varphi$ we obtain

$$
\begin{aligned}
\lambda \int_{\Omega} w_{n} \xi d x+\int_{\Omega} A(x) \nabla w_{n} \nabla \xi d x \leq & \frac{C_{\delta}}{\varepsilon^{q-1}} \int_{\Omega}\left|\nabla w_{n}\right|^{q} \xi d x \\
& +\left\langle I_{n}^{u}, \xi\right\rangle-(1-\varepsilon)\left\langle I_{n}^{v}, \xi\right\rangle,
\end{aligned}
$$


for any $\xi \in C_{c}^{\infty}(\Omega)$. By density, one can allow $\xi=z \psi$ for any $\psi \in C_{c}^{\infty}\left(\mathbf{R}^{N}\right)$ and for any $z \in L^{\infty}(\Omega)$ such that $z \psi \in H_{0}^{1}(\Omega)$.

Now choose $\xi=\left[\left(w_{n}-l\right)^{+}\right]^{2 \bar{q}-1} \psi_{j}^{2}$, where $\psi_{j}=\psi\left(\frac{|x|}{j}\right), \psi \in C_{c}^{\infty}\left(B_{2}\right)$, $\psi \equiv 1$ on $B_{1}$. We get

$$
\begin{aligned}
& \lambda \int_{\Omega} w_{n}\left[\left(w_{n}-l\right)^{+}\right]^{2 \bar{q}-1} \psi_{j}^{2} d x+\alpha(2 \bar{q}-1) \int_{\Omega}\left|\nabla w_{n}\right|^{2}\left[\left(w_{n}-l\right)^{+}\right]^{2 \bar{q}-2} \psi_{j}^{2} d x \\
& \leq \frac{C_{\delta}}{\varepsilon^{q-1}} \int_{\Omega}\left|\nabla w_{n}\right|^{q}\left[\left(w_{n}-l\right)^{+}\right]^{2 \bar{q}-1} \psi_{j}^{2} d x \\
& \quad-2 \int_{\Omega} A(x) \nabla w_{n} \nabla \psi_{j}\left[\left(w_{n}-l\right)^{+}\right]^{2 \bar{q}-1} \psi_{j} d x \\
& \quad+\left\langle I_{n}^{u},\left[\left(w_{n}-l\right)^{+}\right]^{2 \bar{q}-1} \psi_{j}^{2}\right\rangle-(1-\varepsilon)\left\langle I_{n}^{v},\left[\left(w_{n}-l\right)^{+}\right]^{2 \bar{q}-1} \psi_{j}^{2}\right\rangle,
\end{aligned}
$$

Since $\psi_{j} \leq 1$, and due to estimate (2.9) we have

$$
\begin{aligned}
& \left\langle I_{n}^{u},\left[\left(w_{n}-l\right)^{+}\right]^{2 \bar{q}-1} \psi_{j}^{2}\right\rangle-(1-\varepsilon)\left\langle I_{n}^{v},\left[\left(w_{n}-l\right)^{+}\right]^{2 \bar{q}-1} \psi_{j}^{2}\right\rangle \\
& \quad \leq C n^{2 \bar{q}-1}\left[\left\|I_{n}^{u}\right\|_{L^{1}(\Omega)}+\left\|I_{n}^{v}\right\|_{L^{1}(\Omega)}\right]=o(1)_{n} .
\end{aligned}
$$

Using Young's inequality we get

$$
\begin{aligned}
& \lambda \int_{\Omega} w_{n}\left[\left(w_{n}-l\right)^{+}\right]^{2 \bar{q}-1} \psi_{j}^{2} d x \\
& \quad+\frac{\alpha(2 \bar{q}-1)}{2} \int_{\Omega}\left|\nabla w_{n}\right|^{2}\left[\left(w_{n}-l\right)^{+}\right]^{2 \bar{q}-2} \psi_{j}^{2} d x \\
& \leq C_{\delta, \varepsilon} \int_{\Omega}\left[\left(w_{n}-l\right)^{+}\right]^{2 * \bar{q}} \psi_{j}^{2} d x \\
& \quad+C \int_{\Omega}\left|\nabla \psi_{j}\right|^{2}\left[\left(w_{n}-l\right)^{+}\right]^{2 \bar{q}} d x+o(1)_{n} .
\end{aligned}
$$

Observe that $w_{n}$ belongs to $L^{2^{*} \bar{q}}(\Omega)$, since it is so for $u, v$ and $\varphi$. Moreover $\left|\nabla \psi_{j}\right|^{2}$ weakly converges to zero in $L^{\frac{N}{2}}(\Omega)$, so that

$$
\lim _{j \rightarrow+\infty} \int_{\Omega}\left|\nabla \psi_{j}\right|^{2}\left[\left(w_{n}-l\right)^{+}\right]^{2 \bar{q}} d x=0 .
$$

Since (2.36) implies

$$
\begin{aligned}
\left\|\nabla\left(\left[\left(w_{n}-l\right)^{+}\right]^{\bar{q}} \psi_{j}\right)\right\|_{L^{2}(\Omega)}^{2} \leq & C_{\delta, \varepsilon} \int_{\Omega}\left[\left(w_{n}-l\right)^{+}\right]^{2 * \bar{q}} \psi_{j}^{2} d x \\
& +C \int_{\Omega}\left|\nabla \psi_{j}\right|^{2}\left[\left(w_{n}-l\right)^{+}\right]^{2 \bar{q}} d x+o(1)_{n},
\end{aligned}
$$

then passing to the limit as $j$ goes to infinity we find that $\left[\left(w_{n}-l\right)^{+}\right]^{\bar{q}} \in D_{0}^{1,2}(\Omega)$ and

$$
\left\|\left[\left(w_{n}-l\right)^{+}\right]^{\bar{q}}\right\|_{D_{0}^{1,2}(\Omega)}^{2} \leq C_{\delta, \varepsilon} \int_{\Omega}\left[\left(w_{n}-l\right)^{+}\right]^{2^{*} \bar{q}} d x+o(1)_{n} .
$$


Using Sobolev inequality and that

$$
w_{n} \rightarrow u-(1-\varepsilon) v-\varepsilon \varphi \quad \text { strongly in } L^{\bar{q} 2^{*}}(\Omega) \text { as } n \text { tends to infinity, }
$$

letting $n$ go to infinity the conclusion follows as in Theorem 2.4.

Note that when $\lambda>0$ one has that $|u|_{u}^{\bar{q}-1}$ belongs to $H_{0}^{1}(\Omega)$ and not only to $D_{0}^{1,2}(\Omega)$.

Finally, when $\lambda>0$ a similar result can be given in case of Neumann boundary conditions proceeding as in Theorem 2.10.

\section{The case $q \leq 1+\frac{2}{N}$}

We start by extending Theorem 2.4 to the case $q<1+\frac{2}{N}$. However, in view of (2.3) and (1.5), in this case solutions do not belong in general to $H_{0}^{1}(\Omega)$, so that one needs first to define a suitable concept of solution. It seems useful to adopt the notion of renormalized solutions; this notion, introduced first in [12] for transport equations, has been adapted to second order elliptic equations in $[6,19]$, and recently used in several other contexts when dealing with unbounded solutions having infinite energy.

Let us recall that the auxiliary functions $T_{n}(s)$ are defined in (2.6).

Definition 3.1. A renormalized solution of problem (2.1) is a function $u \in L^{1}(\Omega)$ such that $T_{n}(u) \in H_{0}^{1}(\Omega)$ for any $n>0, H(x, \nabla u) \in L^{1}(\Omega)$ and which satisfies

$$
\lambda \int_{\Omega} u S(u) \xi d x+\int_{\Omega} A(x) \nabla u \nabla(S(u) \xi) d x=\int_{\Omega} H(x, \nabla u) S(u) \xi d x
$$

for any Lipschitz function $S$ having compact support and for any $\xi \in H^{1}(\Omega) \cap$ $L^{\infty}(\Omega)$ such that $S(u) \xi \in H_{0}^{1}(\Omega)$.

Renormalized subsolutions or supersolutions are defined in the same way by replacing the equality in (3.1) with the suitable inequality.

Clearly, if $u \in H_{0}^{1}(\Omega)$ is a weak solution then it is also a renormalized solution: indeed, one can choose $S(u) \xi \in H_{0}^{1}(\Omega) \cap L^{\infty}(\Omega)$ as test function in (2.4) and obtain (3.1). Thus, for $H_{0}^{1}(\Omega)$ solutions, the weak and renormalized formulations are equivalent. However, as in the previous section, we will deal here with solutions $u$ such that $(1+|u|)^{\bar{q}-1} u \in H_{0}^{1}(\Omega)$, where $\bar{q}=\frac{(N-2)(q-1)}{2(2-q)}$ : if $q<1+\frac{2}{N}$ then $\bar{q}<1$, so that solutions do not have finite energy (i.e. they are not in $H_{0}^{1}(\Omega)$ ). In this case, the renormalized formulation is meant to allow test functions depending on $u$ itself, which can not be ensured by using the simpler distributional formulation. Another possible formulation based on a duality argument is mentioned later (see (3.26)). 
The existence of a renormalized solution $u$ such that $(1+|u|)^{\bar{q}-1} u \in H_{0}^{1}(\Omega)$ has been proved in [15]. The method of proof given in Section 2 can be easily adapted to provide uniqueness of such solutions.

Theorem 3.2. Assume (1.3), (2.2), (2.3) with $q<1+\frac{2}{N}$. Let $\lambda>0$. If $u$ and $v$ are respectively renormalized subsolution and supersolution of (2.1) such that $(1+|u|)^{\bar{q}-1} u \in H_{0}^{1}(\Omega)$ and $(1+|v|)^{\bar{q}-1} v \in H_{0}^{1}(\Omega)$, then we have $u \leq v$ in $\Omega$.

In particular, problem (2.1) has at most one renormalized solution $u$ such that $(1+|u|)^{\bar{q}-1} u \in H_{0}^{1}(\Omega)$.

Proof. First we observe that Lemma 2.2 still holds for renormalized solutions: indeed, choosing in (3.1) $S=\theta_{n}$ (see (2.6)) yields the same as (2.10), so that we have

$$
\lambda \int_{\Omega} T_{n}(u) \xi d x+\int_{\Omega} A(x) \nabla T_{n}(u) \nabla \xi d x \leq \int_{\Omega} H\left(x, \nabla T_{n}(u)\right) \xi d x+\left\langle I_{n}^{u}, \xi\right\rangle,
$$

where $I_{n}^{u}$ is defined as in (2.8). Moreover, proceeding exactly as in Lemma 2.2 (to be precise, we use that $\left[|\nabla u|^{q}|u|^{2 \bar{q}-1}+f|u|^{2 \bar{q}-1}\right] \chi_{\{|u|>1\}} \in L^{1}(\Omega)$ rather than (2.14) and (2.15)) we obtain the estimate

$$
\lim _{n \rightarrow+\infty} n^{2 \bar{q}-1}\left\|I_{n}^{u}\right\|_{L^{1}(\Omega)}=0 .
$$

The same can be proved as regards $v$. Then, using the convexity of $H$, we follow the proof of Theorem 2.4, in order to obtain that

$$
\begin{aligned}
\lambda \int_{\Omega} & {\left[T_{n}(u)-(1-\varepsilon) T_{n}(v)-\varepsilon \varphi\right] \xi d x } \\
& +\int_{\Omega} A(x) \nabla\left[T_{n}(u)-(1-\varepsilon) T_{n}(v)-\varepsilon \varphi\right] \nabla \xi d x \\
\leq & \frac{C_{\delta}}{\varepsilon^{q-1}} \int_{\Omega}\left|\nabla\left[T_{n}(u)-(1-\varepsilon) T_{n}(v)-\varepsilon \varphi\right]\right|^{q} \xi d x \\
& -\varepsilon\left[\int_{\Omega} \lambda \varphi \xi+A(x) \nabla \varphi \nabla \xi d x-(\gamma+\delta)|\nabla \varphi|^{q} \xi-f(x) \xi\right] \\
& +\left\langle I_{n}^{u}, \xi\right\rangle-(1-\varepsilon)\left\langle I_{n}^{v}, \xi\right\rangle,
\end{aligned}
$$

for any $\varphi, \xi \in H_{0}^{1}(\Omega) \cap L^{\infty}(\Omega), \xi \geq 0$. We define here $\varphi_{n}$ to be a solution of

$$
\begin{cases}\lambda \varphi_{n}-\operatorname{div}\left(A(x) \nabla \varphi_{n}\right)=(\gamma+\delta)\left|\nabla \varphi_{n}\right|^{q}+T_{n}(f(x)) & \text { in } \Omega, \\ \varphi_{n}=0 & \text { on } \partial \Omega .\end{cases}
$$

Note that $\varphi_{n}$ is nonnegative and belongs to $H_{0}^{1}(\Omega) \cap L^{\infty}(\Omega)$. It is proved in [15] that $\left(1+\varphi_{n}\right)^{\bar{q}-1} \varphi_{n}$ is bounded in $H_{0}^{1}(\Omega)$ and

$$
\varphi_{n} \rightarrow \varphi \quad \text { strongly in } L^{\bar{q} 2^{*}}(\Omega),
$$


where $\varphi$ is a renormalized solution of (3.5) corresponding to $f$, and satisfying $(1+$ $\varphi)^{\bar{q}-1} \varphi \in H_{0}^{1}(\Omega)$.

Setting

$$
w_{n}=T_{n}(u)-(1-\varepsilon) T_{n}(v)-\varepsilon \varphi_{n}
$$

and using the equation satisfied by $\varphi_{n}$ we obtain from (3.4)

$$
\begin{gathered}
\lambda \int_{\Omega} w_{n} \xi d x+\int_{\Omega} A(x) \nabla w_{n} \nabla \xi d x \leq \frac{C_{\delta}}{\varepsilon^{q-1}} \int_{\Omega}\left|\nabla w_{n}\right|^{q} \xi d x \\
-\varepsilon \int_{\Omega}\left(T_{n}(f)-f\right) \xi d x+\left\langle I_{n}^{u}, \xi\right\rangle-(1-\varepsilon)\left\langle I_{n}^{v}, \xi\right\rangle .
\end{gathered}
$$

Note that since $\frac{N}{N-1}<q<1+\frac{2}{N}$ then the exponent $\bar{q} \in\left(\frac{1}{2}, 1\right)$, hence $2 \bar{q}-1 \in$ $(0,1)$; for this reason we choose now $\xi=\xi_{n, \sigma}$ with

$$
\xi_{n, \sigma}=\left[\sigma+\left(w_{n}-l\right)^{+}\right]^{2 \bar{q}-1}-\sigma^{2 \bar{q}-1},
$$

where $l, \sigma>0$. We have, using (1.3), that

$$
\liminf _{\sigma \rightarrow 0} \int_{\Omega} A(x) \nabla w_{n} \nabla \xi_{n, \sigma} d x \geq \frac{(2 \bar{q}-1) \alpha}{\bar{q}^{2}} \int_{\Omega}\left|\nabla\left[\left(w_{n}-l\right)^{+}\right]^{\bar{q}}\right|^{2} d x .
$$

Note that $\xi_{n, \sigma} \leq\left[\left(w_{n}-l\right)^{+}\right]^{2 \bar{q}-1}$, and clearly $\xi_{n, \sigma} \rightarrow\left[\left(w_{n}-l\right)^{+}\right]^{2 \bar{q}-1}$ as $\sigma \rightarrow 0$. From (3.7) we obtain, as $\sigma \rightarrow 0$,

$$
\begin{aligned}
\lambda \int_{\Omega} & w_{n}\left[\left(w_{n}-l\right)^{+}\right]^{2 \bar{q}-1} d x+\frac{(2 \bar{q}-1) \alpha}{\bar{q}^{2}} \int_{\Omega}\left|\nabla\left[\left(w_{n}-l\right)^{+}\right]^{\bar{q}}\right|^{2} d x \\
\leq & \frac{C_{\delta}}{\varepsilon^{q-1}} \int_{\Omega}\left|\nabla w_{n}\right|^{q}\left[\left(w_{n}-l\right)^{+}\right]^{2 \bar{q}-1} d x \\
& -\varepsilon \int_{\Omega}\left(T_{n}(f)-f\right)\left[\left(w_{n}-l\right)^{+}\right]^{2 \bar{q}-1} d x \\
& +\left\langle I_{n}^{u},\left[\left(w_{n}-l\right)^{+}\right]^{2 \bar{q}-1}\right\rangle-(1-\varepsilon)\left\langle I_{n}^{v},\left[\left(w_{n}-l\right)^{+}\right]^{2 \bar{q}-1}\right\rangle
\end{aligned}
$$

Since $\left(w_{n}-l\right)^{+} \leq 2 n$, using (3.3) we obtain that last two terms go to zero as $n$ tends to infinity. Moreover, since $u$ and $v$ belong to $L^{\bar{q} 2^{*}}(\Omega)$ and using (3.6), we have that $\left[\left(w_{n}-l\right)^{+}\right]^{2 \bar{q}-1}$ converges strongly in $L^{\frac{\bar{q} 2^{*}}{2 \bar{q}-1}}(\Omega)$; but we have $\frac{\bar{q} 2^{*}}{2 \bar{q}-1}=\left(\frac{N}{q^{\prime}}\right)^{\prime}$, and since $T_{n}(f)-f$ strongly converges to zero in $L^{N / q^{\prime}}(\Omega)$, we conclude that

$$
\begin{aligned}
& \lambda \int_{\Omega} w_{n}\left[\left(w_{n}-l\right)^{+}\right]^{2 \bar{q}-1} d x+\frac{(2 \bar{q}-1) \alpha}{\bar{q}^{2}} \int_{\Omega}\left|\nabla\left[\left(w_{n}-l\right)^{+}\right]^{\bar{q}}\right|^{2} d x \\
& \quad \leq \frac{C_{\delta}}{\varepsilon^{q-1}} \int_{\Omega}\left|\nabla w_{n}\right|^{q}\left[\left(w_{n}-l\right)^{+}\right]^{2 \bar{q}-1} d x+o(1)_{n}
\end{aligned}
$$

where $o(1)_{n}$ goes to zero as $n$ tends to infinity. This inequality is the same as (2.27), and the conclusion of the proof is exactly as in Theorem 2.4. 
A similar result holds in case $\lambda=0$ if the data satisfy a suitable size condition, following the same principle as in Theorem 2.5. We leave the details to the reader.

We are going now to see a different approach to uniqueness, which is based on a simpler linearization principle. This approach, which was not consistent in the situation of Section 2 since it requires too regularity on the solutions. However, it is allowed if $q \leq 1+\frac{2}{N}$ (note that the limiting value $q=1+\frac{2}{N}$ is included too), and provides uniqueness in a more general context. Namely, we consider the problem

$$
\begin{cases}\lambda u-\operatorname{div}(a(x, \nabla u))=H(x, \nabla u) & \text { in } \Omega, \\ u=0 & \text { on } \partial \Omega\end{cases}
$$

where $a(x, \xi): \Omega \times \mathbf{R}^{N} \rightarrow \mathbf{R}^{N}$ is a Carathéodory function such that, for a.e. $x \in \Omega$ and for every $\xi, \eta \in \mathbf{R}^{N}$ :

$$
\begin{gathered}
{[a(x, \xi)-a(x, \eta)] \cdot(\xi-\eta) \geq \alpha|\xi-\eta|^{2}, \quad \alpha>0, \text { and } a(x, 0)=0} \\
|a(x, \xi)| \leq \beta(k(x)+|\xi|), \quad \beta>0, \quad k(x) \in L^{2}(\Omega) .
\end{gathered}
$$

We assume that $H(x, \xi): \Omega \times \mathbf{R}^{N} \rightarrow \mathbf{R}$ is a Carathéodory function which satisfies, for a.e. $x \in \Omega$ and for every $\xi, \eta \in \mathbf{R}^{N}$,

$$
\begin{gathered}
|H(x, \xi)-H(x, \eta)| \leq \gamma\left(b(x)+|\xi|^{q-1}+|\eta|^{q-1}\right)|\xi-\eta|, \\
b(x) \in L^{N}(\Omega), \gamma>0,
\end{gathered}
$$

and

$$
H(x, 0) \in L^{\frac{N}{q^{\prime}}}(\Omega) .
$$

Note that assumptions (3.11) and (3.12) imply that $H(x, \xi)$ satisfies the growth condition (2.3). On the other hand, no convexity is now assumed on $H(x, \cdot)$.

As in Definition 3.1, we say that a function $u \in L^{1}(\Omega)$ is a renormalized subsolution (supersolution) of problem (3.8) if $T_{n}(u) \in H_{0}^{1}(\Omega)$ for any $n>0$, $H(x, \nabla u) \in L^{1}(\Omega)$ and

$$
\lambda \int_{\Omega} u S(u) \xi d x+\int_{\Omega} a(x, \nabla u) \nabla(S(u) \xi) d x \leq(\geq) \int_{\Omega} H(x, \nabla u) S(u) \xi d x
$$

for any Lipschitz function $S$ having compact support and for any $\xi \in H^{1}(\Omega) \cap$ $L^{\infty}(\Omega)$ such that $S(u) \xi \in H_{0}^{1}(\Omega)$, with $S(u) \xi \geq 0$.

We start with two important properties of solutions in the class (1.9). We will need a slight modification of the truncation functions $T_{n}(s)$. Namely, we set

$$
\mathcal{T}_{n}(t)=\int_{0}^{t} \mathcal{T}_{n}^{\prime}(s) d s, \quad \mathcal{T}_{n}^{\prime}(s)= \begin{cases}1 & \text { if }|s|<n \\ n+1-|s| & \text { if } n<|s|<n+1 \\ 0 & \text { if }|s|>n+1\end{cases}
$$


Lemma 3.3. Assume (3.9)-(3.12) with $\frac{N}{N-1}<q \leq 1+\frac{2}{N}$. Let $\lambda \geq 0$. Let $u$ be a renormalized subsolution of (3.8) such that $(1+|u|)^{\bar{q}-1} u \in H_{0}^{1}(\Omega)$, with $\bar{q}=\frac{(N-2)(q-1)}{2(2-q)}$. Then we have

(i)

$$
u \in W_{0}^{1, N(q-1)}(\Omega)
$$

(ii)

$$
\lim _{n \rightarrow+\infty} n^{2 \bar{q}-1} \int_{\{h<u<n+1\}} a(x, \nabla u) \nabla u d x=0 .
$$

(iii) for every nonnegative $\xi \in H_{0}^{1}(\Omega) \cap L^{\infty}(\Omega)$ and for every $n$

$$
\lambda \int_{\Omega} \mathcal{T}_{n}(u) \xi d x+\int_{\Omega} a(x, \nabla u) \mathcal{T}_{n}^{\prime}(u) \nabla \xi d x \leq \int_{\Omega} H\left(x, \nabla \mathcal{T}_{n}(u)\right) \xi d x+\left\langle I_{n}^{u}, \xi\right\rangle,
$$

with

$$
\lim _{n \rightarrow+\infty} n^{2 \bar{q}-1}\left\|I_{n}^{u}\right\|_{L^{1}(\Omega)}=0 .
$$

Proof. The regularity (3.14) follows directly from the fact that $(1+|u|)^{\bar{q}-1} u \in$ $H_{0}^{1}(\Omega)$. This was first observed, in a different context, in [7]; for the reader's convenience, we sketch the simple argument. Indeed, due to Sobolev and Hölder's inequalities, we have

$$
\begin{aligned}
& \left(\int_{\Omega}(|u|)^{(N(q-1))^{*}} d x\right)^{2-q} \leq \int_{\Omega}|\nabla u|^{N(q-1)} d x \\
& \leq\left(\int_{\Omega} \frac{|\nabla u|^{2}}{(1+|u|)^{2-2 \bar{q}}}\right)^{\frac{N(q-1)}{2}}\left(\int_{\Omega}(1+|u|)^{\frac{2 N(q-1)(1-\bar{q})}{2-N(q-1)}} d x\right)^{1-\frac{N(q-1)}{2}} .
\end{aligned}
$$

Since, by definition of $\bar{q}$, we have $\frac{2 N(q-1)(1-\bar{q})}{2-N(q-1)}=(N(q-1))^{*}$ and since $2-q>$ $1-\frac{N(q-1)}{2}$, we conclude that

$$
\|u\|_{W_{0}^{1, N(q-1)}(\Omega)} \leq c\left(1+\left\|(1+|u|)^{\bar{q}-1} u\right\|_{H_{0}^{1}(\Omega)}^{\frac{1}{q}}\right) .
$$

To prove (ii), take in (3.13) $\xi=1$ and $S(t)=\theta_{n}(t) \int_{0}^{t}|s|^{2 \bar{q}-1} \chi_{\{n-1<s<n\}} d s$, where $\theta_{n}$ is defined in (2.6). Since $S(t) \leq c|t|^{2 \bar{q}-1} \chi_{\{n-1<|t|\}}$ we have

$$
\begin{aligned}
\int_{\{n-1<u<n\}} a(x, \nabla u) \nabla u|u|^{2 \bar{q}-1} d x \leq & c \int_{\{n-1<|u|\}}|H(x, \nabla u)||u|^{2 \bar{q}-1} d x \\
& +\frac{c}{n} \int_{\{n<|u|<2 n\}} a(x, \nabla u) \nabla u|u|^{2 \bar{q}-1} d x .
\end{aligned}
$$


Observe that, by (3.10),

$$
\frac{1}{n} \int_{\{n<|u|<2 n\}} a(x, \nabla u) \nabla u|u|^{2 \bar{q}-1} d x \leq c \int_{\{n<|u|<2 n\}}[k(x)+|\nabla u|]|\nabla u||u|^{2 \bar{q}-2} d x
$$

which yields, since $\bar{q} \leq 1$,

$$
\frac{1}{n} \int_{\{n<|u|<2 n\}} a(x, \nabla u) \nabla u|u|^{2 \bar{q}-1} d x \leq c \int_{\{n<|u|<2 n\}}\left[k(x)^{2}+\left|\nabla\left(|u|^{\bar{q}-1} u\right)\right|^{2}\right] d x .
$$

Thus

$$
\lim _{n \rightarrow+\infty} \frac{1}{n} \int_{\{n<|u|<2 n\}} a(x, \nabla u) \nabla u|u|^{2 \bar{q}-1} d x=0
$$

Moreover, since $H$ still satisfies (2.3), we have, as in the proof of Lemma 2.2,

$$
\int_{\{n-1<|u|\}}|H(x, \nabla u)||u|^{2 \bar{q}-1} d x \leq c \int_{\{n-1<|u|\}}\left[\left|\nabla\left(|u|^{\bar{q}-1} u\right)\right|^{2}+\left(|u|^{\bar{q}}\right)^{2^{*}}\right] d x \stackrel{n \rightarrow+\infty}{\longrightarrow} 0
$$

so that we conclude from (3.18)

$$
\int_{\{n-1<u<n\}} a(x, \nabla u) \nabla u|u|^{2 \bar{q}-1} d x \stackrel{n \rightarrow+\infty}{\longrightarrow} 0
$$

hence (3.15).

The proof of (iii) follows the outlines of Lemma 2.2. Choose $S=\mathcal{T}_{n}^{\prime}(t)$ in (3.13), so that

$$
\lambda \int_{\Omega} \mathcal{T}_{n}(u) \xi d x+\int_{\Omega} a(x, \nabla u) \mathcal{T}_{n}^{\prime}(u) \nabla \xi d x \leq \int_{\Omega} H\left(x, \nabla \mathcal{T}_{n}(u)\right) \xi d x+\left\langle I_{n}^{u}, \xi\right\rangle
$$

where $I_{n}^{u}$ is defined as

$$
\begin{aligned}
\left\langle I_{n}^{u}, \xi\right\rangle= & \int_{\{n<u<n+1\}} a(x, \nabla u) \nabla u \xi d x \\
& +\lambda \int_{\Omega}\left(\mathcal{T}_{n}(u)-u \mathcal{T}_{n}^{\prime}(u)\right) \xi d x \\
& +\int_{\Omega}\left(H(x, \nabla u) \mathcal{T}_{n}^{\prime}(u)-H\left(x, \nabla \mathcal{T}_{n}(u)\right)\right) \xi d x
\end{aligned}
$$


As in Lemma 2.2, using the growth condition on $H$ we obtain (2.16), and similarly (2.17); moreover, for the first term of (3.19) we use (3.15). Finally, we can conclude that (3.17) holds.

Note that the borderline value $q=1+\frac{2}{N}$ is included in the previous lemma as well as in the following comparison result. However, some statements would read simpler for this case: in fact, if $q=1+\frac{2}{N}$ then $\bar{q}=1$, hence $(1+|u|)^{\bar{q}-1} u=$ $u$, which belongs to $H_{0}^{1}(\Omega)$ (and (3.14) says the same); in particular, in this case renormalized solutions are also standard $H_{0}^{1}(\Omega)$ weak solutions.

Theorem 3.4. Assume (3.9)-(3.12) with $\frac{N}{N-1}<q \leq 1+\frac{2}{N}$. Let $\lambda \geq 0$. If $u$ and $v$ are respectively a renormalized subsolution and supersolution of (3.8) such that $(1+|u|)^{\bar{q}-1} u \in H_{0}^{1}(\Omega)$ and $(1+|v|)^{\bar{q}-1} v \in H_{0}^{1}(\Omega)$, then we have $u \leq v$ in $\Omega$.

In particular, problem (3.8) has at most one renormalized solution $u$ such that $(1+|u|)^{\bar{q}-1} u \in H_{0}^{1}(\Omega)$.

Proof. From Lemma 3.3 we have that

$$
\begin{aligned}
& \lambda \int_{\Omega} \mathcal{T}_{n}(u) \xi d x+\int_{\Omega} a\left(x, \nabla \mathcal{T}_{n}(u)\right) \nabla \xi d x \leq \int_{\Omega} H\left(x, \nabla \mathcal{T}_{n}(u)\right) \xi d x \\
& +\int_{\Omega}\left[a\left(x, \nabla \mathcal{T}_{n}(u)\right)-a(x, \nabla u) \mathcal{T}_{n}^{\prime}(u)\right] \nabla \xi d x+\left\langle I_{n}^{u}, \xi\right\rangle,
\end{aligned}
$$

for any $\xi \in H_{0}^{1}(\Omega) \cap L^{\infty}(\Omega), \xi \geq 0$, where $I_{n}^{u}$ (defined in (3.19)) satisfies (3.17). Similarly we deal with the equation satisfied by $v$, so that

$$
\begin{aligned}
& \lambda \int_{\Omega} \mathcal{T}_{n}(v) \xi d x+\int_{\Omega} a\left(x, \nabla \mathcal{T}_{n}(v)\right) \nabla \xi d x \geq \int_{\Omega} H\left(x, \nabla \mathcal{T}_{n}(v)\right) \xi d x \\
& +\int_{\Omega}\left[a\left(x, \nabla \mathcal{T}_{n}(v)\right)-a(x, \nabla v) \mathcal{T}_{n}^{\prime}(v)\right] \nabla \xi d x+\left\langle I_{n}^{v}, \xi\right\rangle
\end{aligned}
$$

where

$$
n^{2 \bar{q}-1}\left\|I_{n}^{v}\right\|_{L^{1}(\Omega)} \stackrel{n \rightarrow+\infty}{\rightarrow} 0
$$

For $k>0$, let us set $G_{k}(s)=(s-k)^{+}$: subtracting (3.21) from (3.20) and choosing

$$
\xi=\left[G_{k}\left(\mathcal{T}_{n}(u)-\mathcal{T}_{n}(v)\right)+\sigma\right]^{2 \bar{q}-1}-\sigma^{2 \bar{q}-1}, \quad \sigma>0
$$


we get

$$
\begin{aligned}
& \lambda \int_{\Omega}\left(\mathcal{T}_{n}(u)-\mathcal{T}_{n}(v)\right)\left(\left[G_{k}\left(\mathcal{T}_{n}(u)-\mathcal{T}_{n}(v)\right)+\sigma\right]^{2 \bar{q}-1}-\sigma^{2 \bar{q}-1}\right) d x \\
& +\int_{\Omega}\left[a\left(x, \nabla \mathcal{T}_{n}(u)\right)-a\left(x, \nabla \mathcal{T}_{n}(v)\right)\right] \nabla\left[G_{k}\left(\mathcal{T}_{n}(u)-\mathcal{T}_{n}(v)\right)+\sigma\right]^{2 \bar{q}-1} d x \\
& \leq \int_{\Omega}\left[H\left(x, \nabla \mathcal{T}_{n}(u)\right)-H\left(x, \nabla \mathcal{T}_{n}(v)\right)\right]\left(\left[G_{k}\left(\mathcal{T}_{n}(u)-\mathcal{T}_{n}(v)\right)+\sigma\right]^{2 \bar{q}-1}-\sigma^{2 \bar{q}-1}\right) d x \\
& +\int_{\Omega}\left[a\left(x, \nabla \mathcal{T}_{n}(u)\right)-a(x, \nabla u) \mathcal{T}_{n}^{\prime}(u)\right] \nabla\left[G_{k}\left(\mathcal{T}_{n}(u)-\mathcal{T}_{n}(v)\right)+\sigma\right]^{2 \bar{q}-1} d x \\
& +\int_{\Omega}\left[a\left(x, \nabla \mathcal{T}_{n}(v)\right)-a(x, \nabla v) \mathcal{T}_{n}^{\prime}(v)\right] \nabla\left[G_{k}\left(\mathcal{T}_{n}(u)-\mathcal{T}_{n}(v)\right)+\sigma\right]^{2 \bar{q}-1} d x \\
& +\left\langle\left|I_{n}^{u}\right|,\left[G_{k}\left(\mathcal{T}_{n}(u)-\mathcal{T}_{n}(v)\right)+\sigma\right]^{2 \bar{q}-1}\right\rangle+\left\langle\left|I_{n}^{v}\right|,\left[G_{k}\left(\mathcal{T}_{n}(u)-\mathcal{T}_{n}(v)\right)+\sigma\right]^{2 \bar{q}-1}\right\rangle .
\end{aligned}
$$

Since $\left[G_{k}\left(\mathcal{T}_{n}(u)-\mathcal{T}_{n}(v)\right)\right]^{2 \bar{q}-1} \leq c n^{2 \bar{q}-1}$ last two terms go to zero as $n$ tends to infinity thanks to (3.17) and (3.22). Moreover we have from (3.10)

$$
\begin{aligned}
\left|a\left(x, \nabla \mathcal{T}_{n}(u)\right)-a(x, \nabla u) \mathcal{T}_{n}^{\prime}(u)\right| & \leq\left[\left|a\left(x, \nabla \mathcal{T}_{n}(u)\right)\right|+|a(x, \nabla u)|\right] \chi_{\{n<|u|<n+1\}} \\
& \leq c\left[|\nabla u| \chi_{\{n<|u|<n+1\}}+k(x) \chi_{\{n<|u|<n+1\}}\right] .
\end{aligned}
$$

Using (3.9) and Young's inequality, we have

$$
\begin{aligned}
& \lambda \int_{\Omega}\left(\mathcal{T}_{n}(u)-\mathcal{T}_{n}(v)\right)\left(\left[G_{k}\left(\mathcal{T}_{n}(u)-\mathcal{T}_{n}(v)\right)+\sigma\right]^{2 \bar{q}-1}-\sigma^{2 \bar{q}-1}\right) d x \\
& +\frac{\alpha}{2}(2 \bar{q}-1) \int_{\Omega}\left|\nabla G_{k}\left(\mathcal{T}_{n}(u)-\mathcal{T}_{n}(v)\right)\right|^{2}\left[G_{k}\left(\mathcal{T}_{n}(u)-\mathcal{T}_{n}(v)\right)+\sigma\right]^{2 \bar{q}-2} d x \\
& \leq c \int_{\Omega}\left[H\left(x, \nabla \mathcal{T}_{n}(u)\right)-H\left(x, \nabla \mathcal{T}_{n}(v)\right)\right] \times \\
& \times\left(\left[G_{k}\left(\mathcal{T}_{n}(u)-\mathcal{T}_{n}(v)\right)+\sigma\right]^{2 \bar{q}-1}-\sigma^{2 \bar{q}-1}\right) d x \\
& +c \int_{\Omega}\left[|\nabla u| \chi_{\{n<|u|<n+1\}}+k(x) \chi_{\{n<|u|<n+1\}}\right]^{2}\left[G_{k}\left(\mathcal{T}_{n}(u)-\mathcal{T}_{n}(v)\right)+\sigma\right]^{2 \bar{q}-2} d x \\
& +c \int_{\Omega}\left[|\nabla v| \chi_{\{n<|v|<n+1\}}+k(x) \chi_{\{n<|v|<n+1\}}\right]^{2}\left[G_{k}\left(\mathcal{T}_{n}(u)-\mathcal{T}_{n}(v)\right)+\sigma\right]^{2 \bar{q}-2} d x+o(1)_{n} .
\end{aligned}
$$


Thanks to (3.15) in Lemma 3.3, and since $0<2 \bar{q}-1 \leq 1$, last two terms go to zero as $n$ tends to infinity (for fixed $\sigma>0$ ). Thus, using also that $\lambda \geq 0$ and (3.11) we have

$$
\begin{gathered}
\int_{\Omega}\left|\nabla G_{k}\left(\mathcal{T}_{n}(u)-\mathcal{T}_{n}(v)\right)\right|^{2}\left[G_{k}\left(\mathcal{T}_{n}(u)-\mathcal{T}_{n}(v)\right)+\sigma\right]^{2 \bar{q}-2} d x \\
\leq c \int_{E_{n}}\left[b(x)+\left|\nabla \mathcal{T}_{n}(u)\right|^{q-1}+\left|\nabla \mathcal{T}_{n}(v)\right|^{q-1}\right]\left|\nabla\left(\mathcal{T}_{n}(u)-\mathcal{T}_{n}(v)\right)\right| \times \\
\quad \times\left[G_{k}\left(\mathcal{T}_{n}(u)-\mathcal{T}_{n}(v)\right)+\sigma\right]^{2 \bar{q}-1} d x+o(1)_{n},
\end{gathered}
$$

where

$$
E_{n}=\left\{x: \mathcal{T}_{n}(u)-\mathcal{T}_{n}(v)>k,\left|\nabla\left(\mathcal{T}_{n}(u)-\mathcal{T}_{n}(v)\right)\right|>0\right\} .
$$

Using Young's inequality we get

$$
\begin{aligned}
& \int_{\Omega}\left|\nabla G_{k}\left(\mathcal{T}_{n}(u)-\mathcal{T}_{n}(v)\right)\right|^{2}\left[G_{k}\left(\mathcal{T}_{n}(u)-\mathcal{T}_{n}(v)\right)+\sigma\right]^{2 \bar{q}-2} d x \\
& \leq c \int_{E_{n}}\left[b(x)+\left|\nabla \mathcal{T}_{n}(u)\right|^{q-1}+\left|\nabla \mathcal{T}_{n}(v)\right|^{q-1}\right]^{2} \times \\
& \quad \times\left[G_{k}\left(\mathcal{T}_{n}(u)-\mathcal{T}_{n}(v)\right)+\sigma\right]^{2 \bar{q}} d x+o(1)_{n} .
\end{aligned}
$$

Using Sobolev inequality and that $u, v \in W_{0}^{1, N(q-1)}(\Omega)$, we deduce

$$
\begin{aligned}
& \left(\int_{\Omega}\left(\left[G_{k}\left(\mathcal{T}_{n}(u)-\mathcal{T}_{n}(v)\right)+\sigma\right]^{\bar{q}}-\sigma^{\bar{q}}\right)^{2^{*}} d x\right)^{\frac{2}{2^{*}}} \\
& \leq c\left(\int_{E_{n}}\left[b(x)+|\nabla u|^{q-1}+|\nabla v|^{q-1}\right]^{N} d x\right)^{\frac{2}{N}} \\
& \quad \times\left(\int_{\Omega}\left[G_{k}\left(\mathcal{T}_{n}(u)-\mathcal{T}_{n}(v)\right)+\sigma\right]^{\bar{q} 2^{*}} d x\right)^{\frac{2}{2^{*}}}+o(1)_{n} .
\end{aligned}
$$

Letting $n$ tend to infinity we obtain

$$
\begin{aligned}
& \left(\int_{\Omega}\left(\left[G_{k}(u-v)+\sigma\right]^{\bar{q}}-\sigma^{\bar{q}}\right)^{2^{*}} d x\right)^{\frac{2}{2^{*}}} \leq \\
& \quad \leq c\left(\int_{\langle u-v>k,|\nabla(u-v)|>0\}}\left[b(x)+|\nabla u|^{q-1}+|\nabla v|^{q-1}\right]^{N} d x\right)^{\frac{2}{N}} \\
& \times\left(\int_{\Omega}\left[G_{k}(u-v)+\sigma\right]^{\bar{q} 2^{*}} d x\right)^{\frac{2}{2^{*}}},
\end{aligned}
$$


and then, as $\sigma \rightarrow 0$,

$$
\begin{aligned}
& \left(\int_{\Omega}\left(\left[G_{k}(u-v)\right]^{\bar{q}}\right)^{2^{*}} d x\right)^{\frac{2}{2^{*}}} \\
& \leq C\left(\int_{(u-v>k,|\nabla(u-v)|>0\}}\left[b(x)+|\nabla u|^{q-1}+|\nabla v|^{q-1}\right]^{N} d x\right)^{\frac{2}{N}} \\
& \quad \times\left(\int_{\Omega}\left[G_{k}(u-v)\right]^{\bar{q} 2^{*}} d x\right)^{\frac{2}{2^{*}}} .
\end{aligned}
$$

From this inequality one can deduce that $u \leq v$ in $\Omega$. Indeed, we argue by contradiction. Set $M=\sup (u-v)$; then, should $M$ be positive, even possibly infinite, we have

$$
\lim _{k \rightarrow M} \operatorname{meas}\{x: u-v>k,|\nabla(u-v)|>0\}=0,
$$

since either $M=+\infty$ or $|\nabla(u-v)=0|$ a.e. on $(u-v)=M$. Therefore, using that $u, v \in W_{0}^{1, N(q-1)}(\Omega)$, there exists $k_{0}<M$ such that

$$
\left(\int_{\left\langle u-v>k_{0},|\nabla(u-v)|>0\right\}}\left[b(x)+|\nabla u|^{q-1}+|\nabla v|^{q-1}\right]^{N} d x\right)^{\frac{2}{N}}<\frac{1}{C}
$$

and then (3.25) implies that $(u-v) \leq k_{0}$ almost everywhere, getting a contradiction with the fact that $k_{0}<\sup (u-v)$. We conclude that $u \leq v$.

We point out that the previous theorem extends the uniqueness result which is proved in [5] assuming $H(x, 0) \in H^{-1}(\Omega)$ and for solutions in $H_{0}^{1}(\Omega)$. Let us recall that the existence of $H_{0}^{1}(\Omega)$ solutions can not be proved, nor it is expected to hold, under assumption (3.12) with $q<1+\frac{2}{N}$, so that, to be consistent with the existence results (see [15]) one actually needs to work with solutions in the class (1.9).

\subsection{Comments and remarks}

\section{The formulation by duality in the linear case}

Consider problem (2.1), where the second-order operator is linear. In order to deal with solutions with possibly infinite energy, instead of using the notion of renormalized solution, a different formulation can be given by using the linear character of the operator. 
Definition 3.5 (see [20]). A function $u \in L^{1}(\Omega)$ is a solution of (2.1) if $H(x, \nabla u) \in L^{1}(\Omega)$ and

$$
\lambda \int_{\Omega} u \varphi d x-\int_{\Omega} u \operatorname{div}\left(A^{*}(x) \nabla \varphi\right) d x=\int_{\Omega} H(x, \nabla u) \varphi d x,
$$

for every $\varphi \in H_{0}^{1}(\Omega): \operatorname{div}\left(A^{*}(x) \nabla \varphi\right) \in L^{\infty}(\Omega)$,

where $A^{*}(x)$ denotes the adjoint matrix of $A(x)$.

Note that in Definition 3.5 only a minimal regularity is asked on $u$, by using the advantage of linearity to integrate twice by parts. It is well known (see e.g. [11]) that, since $H(x, \nabla u) \in L^{1}(\Omega)$, any solution in the sense of Definition 3.5 also satisfies the renormalized formulation (3.1). We deduce then the following

Theorem 3.6. Assume (1.3), (3.11), (3.12) with $\frac{N}{N-1}<q \leq 1+\frac{2}{N}$. Let $\lambda \geq 0$. Then there exists at most one function $u$ which is solution of (2.1) in the sense of Definition 3.5 and such that $(1+|u|)^{\bar{q}-1} u \in H_{0}^{1}(\Omega)$, with $\bar{q}=\frac{(N-2)(q-1)}{2(2-q)}$.

A similar result can be given in the convex case (i.e. assuming (2.2) and (2.3)) for the whole range of $q$ such that $\frac{N}{N-1}<q<2$, since the results of Theorem 2.4 and Theorem 3.2 apply to solutions in the sense (3.26) which belong to the class (1.9).

\section{The case $q \leq \frac{N}{N-1}$ and measure data}

The question of finding an appropriate class of solutions where uniqueness holds is not relevant if $q<\frac{N}{N-1}$ (note that the counterexample given in (1.7) holds only for $\left.q>\frac{N}{N-1}\right)$. Indeed, asking only $H(x, \nabla u) \in L^{1}(\Omega)$, the solutions of (2.1) are expected to belong to $W_{0}^{1, r}(\Omega)$ for any $r<\frac{N}{N-1}$, in particular they already satisfy (1.8). In fact, uniqueness results when $q<\frac{N}{N-1}$ have already been proved, see e.g. [4] for a result in a general context including nonlinear operators.

When $q<\frac{N}{N-1}$ and in case of linear operators, one can even prove uniqueness if data are bounded measures, using the formulation (3.26) and a simple duality argument. This was done in [1] for the Laplace operator, for completeness we sketch the result for the general case.

Let $H(x, \xi)$ satisfy

$$
\begin{aligned}
& |H(x, \xi)-H(x, \eta)| \leq \gamma\left(b(x)+|\xi|^{q-1}+|\eta|^{q-1}\right)|\xi-\eta| \\
& \text { with } q<\frac{N}{N-1}, b(x) \in L^{r}(\Omega) \text { for some } r>N, \gamma>0
\end{aligned}
$$

and

$$
H(x, 0) \in L^{1}(\Omega)
$$


Let $\mu$ be a bounded Radon measure in $\Omega$. We say that $u$ is a solution of

$$
\begin{cases}\lambda u-\operatorname{div}(A(x) \nabla u)=H(x, \nabla u)+\mu & \text { in } \Omega, \\ u=0 & \text { on } \partial \Omega,\end{cases}
$$

if $u \in L^{1}(\Omega), H(x, \nabla u) \in L^{1}(\Omega)$ and

$$
\lambda \int_{\Omega} u \varphi d x-\int_{\Omega} u \operatorname{div}\left(A^{*}(x) \nabla \varphi\right) d x=\int_{\Omega} H(x, \nabla u) \varphi d x+\int_{\Omega} \varphi d \mu,
$$

for every $\varphi \in H_{0}^{1}(\Omega): \operatorname{div}\left(A^{*}(x) \nabla \varphi\right) \in L^{\infty}(\Omega)$.

Note that such test functions $\varphi$ are Hölder continuous by means of De GiorgiNash's results, hence they can be tested against measures. Then we have:

Theorem 3.7. Assume (1.3), (3.27) and (3.28), and let $\lambda \geq 0$. Let $\mu$ be a bounded Radon measure in $\Omega$. Then there exists at most one solution u of (3.29).

Proof. Let $u_{i}, i=1,2$, be two solutions of (3.29) in the sense of (3.30). It is known that $u_{i} \in W_{0}^{1, r}(\Omega)$ for any $r<\frac{N}{N-1}$. Moreover, if $H_{n}(x, \xi)$ is a sequence of bounded functions such that $H_{n}\left(x, \nabla u_{i}\right)$ converges to $H\left(x, \nabla u_{i}\right)$ in $L^{1}(\Omega)$, and if $\mu_{n}$ is a sequence of smooth functions converging to $\mu$ in the weak- $*$ topology of measures, then the solutions of

$$
\begin{cases}\lambda u_{i, n}-\operatorname{div}\left(A(x) \nabla u_{i, n}\right)=H_{n}\left(x, \nabla u_{i}\right)+\mu_{n} & \text { in } \Omega, \\ u_{i, n}=0 & \text { on } \partial \Omega\end{cases}
$$

converge to $u_{i}$ in $W_{0}^{1, r}(\Omega)$ for any $r<\frac{N}{N-1}$. Furthermore, one can choose $H_{n}$ to be $C^{1}$ and still satisfying (3.27), and converging to $H(x, \xi)$ locally uniformly. Now, since $u_{i, n}$ belong to $H_{0}^{1}(\Omega) \cap L^{\infty}(\Omega)$, we have

$$
\begin{aligned}
& \int_{\Omega}\left(u_{1, n}-u_{2, n}\right)\left[\lambda \varphi-\operatorname{div}\left(A^{*} \nabla \varphi\right)\right] d x \\
& =\int_{\Omega} \varphi\left[\int_{0}^{1} \frac{\partial H_{n}}{\partial \xi}\left(x, t \nabla u_{1, n}+(1-t) \nabla u_{2, n}\right) d t\right] \nabla\left(u_{1, n}-u_{2, n}\right) d x \\
& \quad+\int_{\Omega}\left[H_{n}\left(x, \nabla u_{1}\right)-H_{n}\left(x, \nabla u_{1, n}\right)\right] \varphi d x \\
& -\int_{\Omega}\left[H_{n}\left(x, \nabla u_{2}\right)-H_{n}\left(x, \nabla u_{2, n}\right)\right] \varphi d x .
\end{aligned}
$$


Set

$$
p_{n}(x)=\int_{0}^{1} \frac{\partial H_{n}}{\partial \xi}\left(x, t \nabla u_{1, n}+(1-t) \nabla u_{2, n}\right) d t
$$

and take $\varphi=\varphi_{n}$ the solution of

$$
\left\{\begin{array}{lr}
\lambda \varphi_{n}-\operatorname{div}\left(A^{*}(x) \nabla \varphi_{n}\right)=-\operatorname{div}\left(p_{n}(x) \varphi_{n}\right)+T_{1}\left(u_{1}-u_{2}\right) & \text { in } \Omega, \\
\varphi_{n}=0 & \text { on } \partial \Omega
\end{array}\right.
$$

Since $H_{n}$ satisfies (3.27)-(3.28), and using that $\nabla u_{i, n}$ strongly converge in $W_{0}^{1, r}(\Omega)$ for every $r<\frac{N}{N-1}$, we have that $H_{n}\left(x, \nabla u_{i, n}\right)$ strongly converges to $H\left(x, \nabla u_{i}\right)$ in $L^{1}(\Omega)$, and there exists $\delta>0$ such that $p_{n}$ is bounded in $L^{N+\delta}(\Omega)^{N}$. By standard regularity results this implies that $\varphi_{n}$ is uniformly bounded in $L^{\infty}(\Omega)$, hence last two terms in (3.32) converge to zero. Passing to the limit we get

$$
\int_{\Omega}\left(u_{1}-u_{2}\right) T_{1}\left(u_{1}-u_{2}\right) d x=0
$$

so that $u_{1}=u_{2}$.

Remark 3.8. Note that the case $N=2$ also enters in the previous situation; indeed, when $N=2$ the values $\frac{N}{N-1}$ and $1+\frac{2}{N}$ coincide and $\frac{N}{N-1}=1+\frac{2}{N}=$ 2. Thus, in the subcritical case $q<2$ the main uniqueness result reads as in Theorem 3.7, at least for linear operators. For nonlinear operators and with data in $L^{1}(\Omega)$, this case is included in the results in [4].

Remark 3.9. Finally, the case $q=\frac{N}{N-1}$ is a critical one; adapting the counterexample (1.7) it is still possible to construct a non trivial solution $u$ of the homogeneous equation

$$
-\Delta u=|\nabla u|^{\frac{N}{N-1}}, \quad u \in W_{0}^{1, \frac{N}{N-1}}(\Omega),
$$

so that looking for a smaller class where uniqueness holds is still necessary. The radial case suggests that uniqueness holds here for solutions $u$ such that $|\nabla u| \in L^{\frac{N}{N-1}}(\log L)^{N-1}$. Indeed, in order to extend Theorem 3.4 to $q=\frac{N}{N-1}$ one should work in the context of Orlicz spaces, and assumptions (3.11)-(3.12) should be suitably modified as well, e.g. by asking $b(x) \in L^{N}(\log L)^{N-1}$ in (3.11) and $H(x, 0) \in L^{1}(\log L)^{N-1}$. 


\section{References}

[1] N. AlAA and M. PIERrE, Weak solutions of some quasilinear elliptic equations with data measures, SIAM J. Math. Anal. 24 (1993), 23-35.

[2] G. BARLES and F. MURAT, Uniqueness and the maximum principle for quasilinear elliptic equations with quadratic growth conditions, Arch. Ration. Mech. Anal. 133 (1995), 77-101.

[3] G. Barles, A-P. Blanc, C. Georgelin and M. Kobylanski, Remarks on the maximum principle for nonlinear elliptic PDEs with quadratic growth conditions, Ann. Scuola Norm. Sup. Pisa Cl. Sci. (4) 28 (1999), 381-404.

[4] F. Betta, A. Mercaldo, F. Murat, M. Porzio, Uniqueness of renormalized solutions to nonlinear elliptic equations with a lower order term and right-hand side in $L^{1}(\Omega)$. A tribute to J. L. Lions, ESAIM Control Optim. Calc. Var. 8 (2002), 239-272.

[5] F. Betta, A. Mercaldo, F. Murat and M. Porzio, Uniqueness results for nonlinear elliptic equations with a lower order term, Nonlinear Anal. 63 (2005), 153-170.

[6] L. Boccardo, I. DiAz, D. GiachetTI and F. Murat, Existence and regularity of renormalized solutions for some elliptic problems involving derivatives of nonlinear terms, J. Differential Equations 106 (1993), 215-237.

[7] L. BOCCARDo and T. GALlOUËT, Nonlinear elliptic equations with right hand side measures, Comm. Partial Differential Equations 17 (1992), 641-655.

[8] L. BocCARdo, F. MURAT and J.P. PUEL, $L^{\infty}$ estimate for some nonlinear elliptic partial differential equations and application to an existence result, SIAM J. Math. Anal. 23 (1992), 326-333.

[9] L. Boccardo, F. Murat and J.P. Puel, Existence de solutions faibles pour des équations elliptiques quasi-linèaires à croissance quadratique, In: "Nonlinear Partial Differential Equations and their Applications". College de France Seminar, Vol. IV (Paris, 1981/1982), Res. Notes in Math. 84, Pitman, Boston, Mass. - London, 1983, 19-73.

[10] A. Dall' Aglio, D. Giachetti and J.P. Puel, Nonlinear elliptic equations with natural growth in general domains, Ann. Mat. Pura Appl. (4) 181 (2002), 407-426.

[11] G. Dal Maso, F. Murat, L. Orsina and A. Prignet, Renormalized solutions of elliptic equations with general measure data, Ann. Scuola Norm. Sup. Pisa Cl. Sci. (4) 28 (1999), 741-808.

[12] A. Di PERnA and P. L. LiOns, On the Cauchy problem for Boltzmann equations: global existence and weak stability, Ann. of Math. (2) 130 (1989), 321-366.

[13] E. FERONE and F. MURAT, Quasilinear problems having quadratic growth in the gradient: an existence result when the source term is small, In: "Equations aux Dérivées Partielles et Applications", Gauthier-Villars, Ed. Sci. Méd. Elsevier, Paris, 1998, 497-515.

[14] N. Grenon, F. Murat and A. Porretta, Existence and a priori estimate for elliptic problems with subquadratic gradient dependent terms, C. R. Acad. Sci. Paris, Ser. I 342 (2006), 23-28.

[15] N. Grenon, F. Murat and A. Porretta, Elliptic equations with superlinear gradient dependent terms, in preparation.

[16] K. HAnsson, V. MAZ'JA and I. E. Verbitsky, Criteria of solvability for multidimensional Riccati equations, Ark. Mat. 37 (1999), 87-120.

[17] P. L. Lions, Résolution de problèmes elliptiques quasilinèaires, Arch. Ration. Mech. Anal. 74 (1980), 335-353.

[18] P. L. Lions, Quelques remarques sur les problèmes elliptiques quasilinèaires du second ordre, J. Anal. Math. 45 (1985), 234-254.

[19] P. L. LiOnS and F. MuRat, Solutions renormalisées d'équations elliptiques non linéaires, unpublished paper. 
[20] G. StAmPaCCHIA, Le problème de Dirichlet pour les équations elliptiques du second ordre à coefficients discontinus, Ann. Inst. Fourier (Grenoble) 15 (1965), 189-258.

Laboratoire de Mathématiques et Physique Théorique

UMR CNRS 6083

Faculté des Sciences et Techniques

Université de Tours

Parc de Grandmont

Tours 37200, France

barles@lmpt.univ-tours.fr

Dipartimento di Matematica

Università di Roma Tor Vergata

Via della Ricerca Scientifica, 1

00133 Roma, Italia

porretta@mat.uniroma2.it 